\title{
Revisiting the mechanisms of oil uptake during deep-frying
}

\author{
Maxime Touffet, Gilles Trystram, Olivier Vitrac* \\ UMR 1145 Food Processing and Engineering, INRA, AgroParisTech \\ Université Paris-Saclay, 91300 Massy, France
}

\section{Highlights}

- Oil penetrates in parfried frozen products during the first minute of deep-frying, and during cooling, once the product is removed from the oil bath.

- The mechanisms of oil penetration during frying involves a Carnot cycle mediated by steam between the crust and the frozen core.

- All regions connected by fissures are accessible to oil penetration during deep-frying regardless of their water content.

- The deep penetration of oil during cooling and immersion stages are under enthalpic control and requires steam condensation.

- Surface oil penetration is conversely under entropic control and is possible only when air (non-condensable phase) can be displaced by oil.

\begin{abstract}
Deep-frying is one of the most used and versatile technique for cooking foods. During immersion stage, it has been well accepted that the internal vaporization created an overpressure across the crust higher than the oil capillary pressure. As a result, oil could penetrate inside the food product only during the very last stages of frying or during cooling. This study shows a
\end{abstract}

\footnotetext{
*Correspondence: olivier.vitrac@agroparistech.fr
} 
more complex picture in parfried frozen French fries with oil being capable of penetrating deep inside the product during the first minute of immersion. The new mechanism invokes a Carnot cycle between hygroscopic and frozen regions mediated by steam along cracks and fissures. Oil labeling and microscopic analysis show that both enthalpic and entropic forces drive oil transport and that oil can replace or be replaced by another phase: new oil, water, air. This work suggests new strategies based on thermodynamics to minimize oil pickup in fried products.

\section{Keywords}

Frying, oil uptake, porous media, coupled heat and mass transfer, modeling

\section{Introduction}

Deep-frying products have considerable importance in the diet of consumers all around the world (Drewnowski and Popkin, 1997). French-fries dominate in western-style convenience foods. Parfried frozen French-fries are thus served in numerous conventional and quick service restaurants, school canteens and are also available for home-made preparation. Due to overweight and obesity concerns, oil uptake in fried products motivated a large number of studies aiming at understanding the mechanisms of oil uptake and at elucidating the relationships between the process conditions and the final oil content in finished products (see significant reviews (Dana and Saguy, 2006; Mellema, 2003; Ziaiifar et al., 2008)). Although deep-frying is one of the oldest cooking operations, it has been only latterly recognized that a significant internal overpressure exceeding the oil capillary pressure prevents oil from penetrating inside the product during the immersion stage (Sandhu et al., 2013; Vitrac et al., 2000). Oil infiltrates the crust only during cooling after the depletion or inversion of the pressure gradient either by spontaneous (Bouchon and Pyle, 2005a, b) and forced imbibition (Vitrac et al., 2000). More recently, the frying process motivated different categories of works, 
where the details of the complex physics of the deep-frying unit operation are the core of the studies. Several phenomena are indeed specific to the operation during immersion stage and are particularly difficult to capture and parameterize in mechanistic models. They include high heat flux densities at the oil-food interface (Vitrac and Trystram, 2005), unsaturated transports of miscible phases (air-vapor, steam-liquid water) and of immiscible ones (steam-oil, air-oil) inside the product, brutal physicochemical changes (starch melting, glass transition induced by drying) inside the product (Aguilera et al., 2001), propagation of mechanical fractures (Pedreschi, 2012).

Early attempts of physical simulation of mass transfer during deep-frying (Halder et al., 2007a, b; Ni and Datta, 1999) were carried out with local thermodynamical equilibrium and continuum mechanics assumptions. The mechanisms of oil adhesion and imbibition were either not considered at all or coarsely represented with a diffusive origin. Such simulations do not reproduce the experimental penetration depth of oil, which is typically limited from two to four cell layers (Achir et al., 2010). Similarly, the competition between oil and air transport to equilibrate the pressure inside the crust and deep inside the product cannot be captured with homogenized models. Detailed experiments showed that cellular defects with coordination numbers strictly larger than two (e.g., "Y" or "X" shapes) cause, indeed, complex displacements of oil and air phases (Cortés et al., 2016; Patsioura et al., 2015). As an illustration, the oil front is accelerated along the corners of parenchyma cells (acute angles) and usually reaches restrictions between cells faster than air. The mechanism coined "gas bubble snap-off" traps air bubbles, which subsequently stop any further oil imbibition (Vauvre et al., 2015). In some respects, hybrid mixture theory (HMT) introduced initially for describing swelling systems (Bennethum and Cushman, 1996) offers an appealing alternative to volume averaging methods in the sense of Whitaker (Whitaker, 1977). The HMT method upscales twice the phenomena from the microscopic (phase level) to mesoscopic scale (equilibrium between phases) and from 
the mesoscopic to macroscopic scale (continuous medium). The hierarchy between scales captures implicitly the effects occurring at microscopic scale (oscillating and immiscible behaviors, saturation). In a recent paper, Sandhu and Takhar (2018) argued that oil penetrates inside the product also during the first minute of deep-frying (see Figure 8 and 10 herein). The calculated penetration depth was commensurable to two cell layers and was representing onethird of the final oil content, while the other two-thirds were associated with oil adhering to the external surface. Similar conclusions have been already reported by us in parfried frozen French-fries using a special dyeing technique (Patsioura et al., 2016; Vauvre et al., 2015). Oil was shown to penetrate during the first minute and to accumulate randomly below the crust and in large defects. In some samples, oil reached the center of the product already filled with water. The reported penetration depths, the size and the distribution of oil spots differed, however, dramatically from previous HMT predictions. The current study addresses the limitations of current mass transport models by analyzing the consequences of mass transfer between the six phases (solid content, ice, liquid water, steam, oil, air) met in parfried frozen products, as well as the role of fractures and their expansion during frying. Beyond its application for future sophistication of deep-frying models, the presented work encourages the development of a mesoscale science (Li et al., 2016a; Li et al., 2016b) to understand and to control oil uptake.

The manuscript is organized as follows. The second section details the experimental conditions to evidence and quantifies oil uptake at different scales (product, tissue and cellular scales) during immersion stage and after full frying. The ad-hoc methodology enables us to relate when the oil migrated inside a specific region of the potato strip (ends, edges, faces, and core). The causality of oil penetration during frying is analyzed and discussed in the third section. Three "mesoscale" levels are considered: i) the scale of pretreatments creating the superstructure (product scale and below), where coupled heat and mass transfer occurs during frying; ii) the scale controlling the heterogeneities of the pressure field inside the product and 
finally (several millimeters), iii) the scale associated to the preferential pathways for oil penetration (few micrometers). The main findings are summarized in the last section. A revised description of oil uptake and new strategies to control it are suggested.

\section{Materials and Methods}

\subsection{Potato French fries processing}

Oil uptake was studied in parfried frozen French fries (prepared at industrial scale from Solanum tuberosum cv. Bintje and stored one month at $-20^{\circ} \mathrm{C}$; supplier McCain, France), and in strips of fresh potatoes (prepared at laboratory scale from Solanum tuberosum cv. Caesar; purchased from local market). Fresh potato samples were peeled and cut into strip shape with dimension $70 \times 9 \times 9 \mathrm{~mm}^{3}$. Parfried frozen French fries with the same dimensions were selected. Raw potato products were processed in several steps to generate samples with different initial states before finish frying, denoted fresh, stored-frozen, parfried-frozen, parfried-stored-frozen, parfried-stored-frozen-thawed. The corresponding processing steps and coding are summarized in Figure 1, with operation conditions detailed in Table 1. Samples coded A-E were processed in the laboratory. Samples D were parfried in a household deep-fryer (model F40-A, 3L, SEB, France) by immersion in sunflower oil (supplier Lesieur, Dunkerque, France) at $180^{\circ} \mathrm{C}$ for one minute. Then, samples B-D were frozen for 24 hours at $-20^{\circ} \mathrm{C}$. Samples coded C and E were stored frozen for 72 hours at temperatures fluctuating around $-10^{\circ} \mathrm{C}$ to reproduce common temperature variations met during distribution and retailing (Gormley et al., 2002). Temperature variations accelerate, indeed, Oswald ripening, crystal growth and consequently stress damage, as shown by Ullah et al. (2014). Finally, all samples were finish fried for five minutes at $180^{\circ} \mathrm{C}$. Samples coded $\mathrm{E}^{*}-\mathrm{G}^{*}$ were produced at industrial scale, and only thawing 
and the finish-frying steps were performed in the laboratory. All experiments were at least triplicated, the number of analyzed sticks is indicated for each condition between parentheses: $\mathrm{A}(11), \mathrm{B}(10), \mathrm{C}(5), \mathrm{D}(12), \mathrm{E}(15), \mathrm{E}^{*}(83), \mathrm{F}^{*}(8), \mathrm{G}^{*}(7)$.

Table 1: Description of production steps described in Figure 1

\begin{tabular}{|c|c|c|c|}
\hline \multirow{2}{*}{ Step } & \multicolumn{2}{|r|}{ Description } & \multirow{2}{*}{ Comments } \\
\hline & Time & Temperature & \\
\hline Cutting & - & $25^{\circ} \mathrm{C}$ & Potato strip $9 \times 9 \times 70 \mathrm{~mm}^{3}$ \\
\hline Par-frying & $1 \mathrm{~min}$ & $180^{\circ} \mathrm{C} \pm 1^{\circ} \mathrm{C}$ & - \\
\hline Freezing & $24 \mathrm{~h}$ & $-20^{\circ} \mathrm{C}$ & - \\
\hline Storage & $72 \mathrm{~h}$ & $\begin{array}{c}\text { Three cycles of temperature } \\
\text { variations ( } 4 \text { hours at }-2^{\circ} \mathrm{C} \text { and } \\
20 \text { hours at }-20^{\circ} \mathrm{C} \text { ) }\end{array}$ & $\begin{array}{l}\text { Methodology similar to the } \\
\text { one used by Gormley et al. } \\
\text { (2002) }\end{array}$ \\
\hline \multirow{2}{*}{ Thawing } & $10 \min$ & $25^{\circ} \mathrm{C}$ & $\begin{array}{l}\text { Final core temperature - } \\
2^{\circ} \mathrm{C}\end{array}$ \\
\hline & $120 \mathrm{~min}$ & $25^{\circ} \mathrm{C}$ & $\begin{array}{l}\text { Final core temperature } \\
20^{\circ} \mathrm{C}\end{array}$ \\
\hline $\begin{array}{l}\text { Finish- } \\
\text { frying }\end{array}$ & $5 \mathrm{~min}$ & $180^{\circ} \mathrm{C} \pm 1^{\circ} \mathrm{C}$ & $\begin{array}{l}\text { Once removed from the } \\
\text { bath, they were dripped } \\
\text { vertically over the bath for } \\
20 \mathrm{~s} \text { (no vibration) }\end{array}$ \\
\hline
\end{tabular}




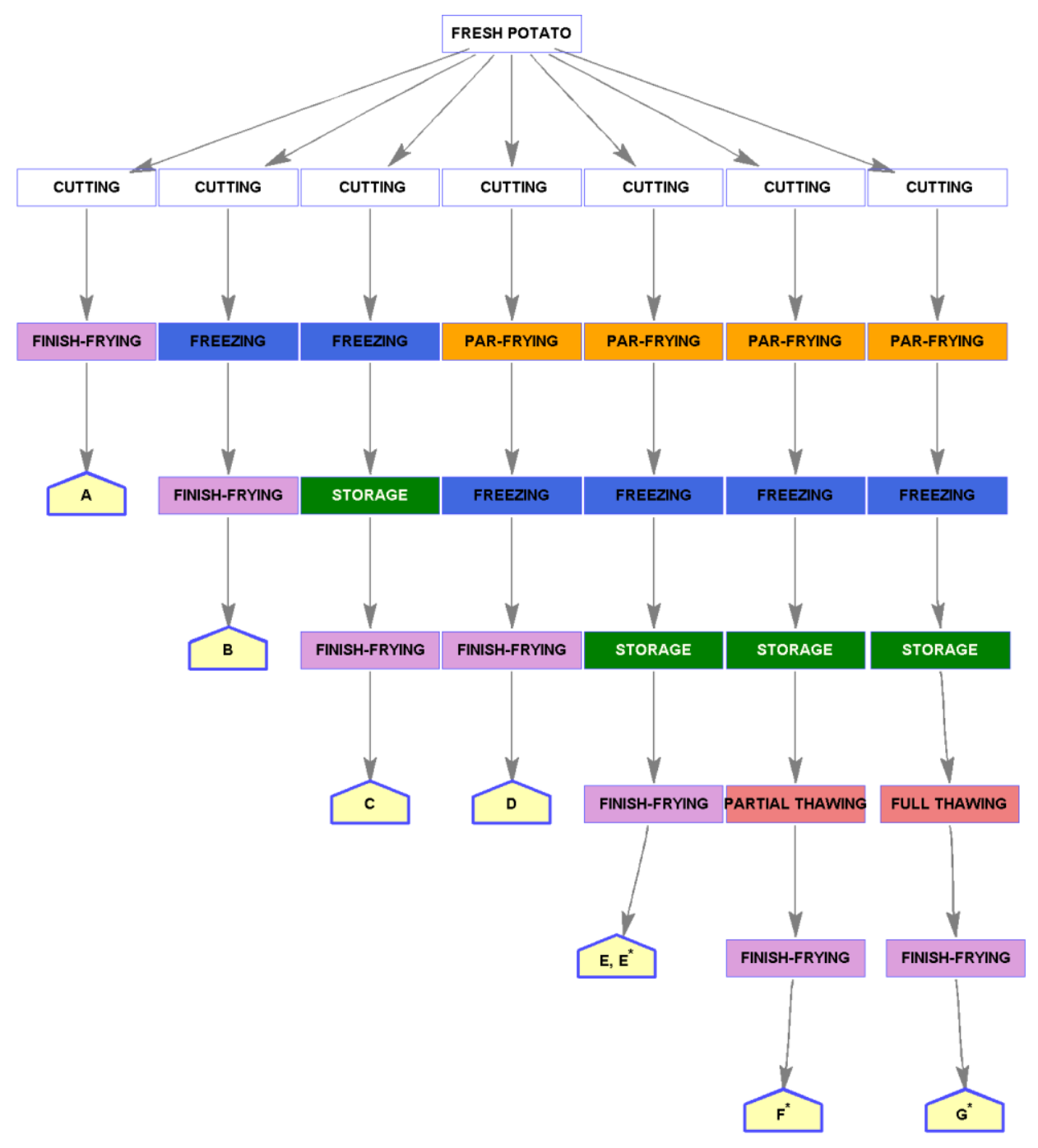

Figure 1: Sample production steps. Samples with stars (*) were produced at industrial scale (from cutting to storage steps). Other samples were produced at laboratory scale (from cutting to finish-frying steps). 


\subsection{Oil distribution and temporal labeling}

The different forms of non-saturated flows in French-fries are particularly challenging to characterize. Oil can penetrate indeed during immersion stage and when the product exposed to air. Oil is already present in parfried products and can be exchanged or replaced by the oil of the finished frying bath. Different strategies were combined to separate parfrying and finished frying oils, surface, and internal oil populations, and to identify when oil penetrates inside the product. The concomitant local dehydration, shrinkage and changes in structure during deepfrying complicates the analysis. To close mass balance, all results (local, averaged over one or several potato strips) were expressed respectively to the local solid mass reference frame.

Temporal labeling. Oil penetrating during the first minute and the remaining time of full frying were identified by using a dye-labeling technique already described by Patsioura et al. (2016) and summarized in Figure 2. The product was successively full fried in two oil baths for a total duration of five minutes. The first minute of full-frying was carried in dark blue oil (oil dyed with $2 \mathrm{~g} / \mathrm{kg}$ of Sudan Blue II - 1,4-Bis(butylamino)-9,10-anthraquinone, CAS number 1735414-2). The product was subsequently transferred to a second bath filled with dark red oil (oil dyed with 2 g/kg of Sudan Red G, 1-(2-Methoxyphenylazo)-2-naphthol, CAS number 122955-6). To reduce the contamination of the red bath by the blue oil adhering to the product, a sharp shake was applied before transfer to detach the excess of blue oil. The transfer time from the blue to the red bath was about $2 \mathrm{~s}$. It was verified that increasing the time did not modify significantly the amount of blue oil in particular in the core region. 


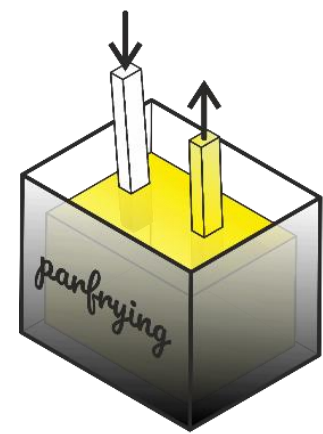

$\sim 1 \min$
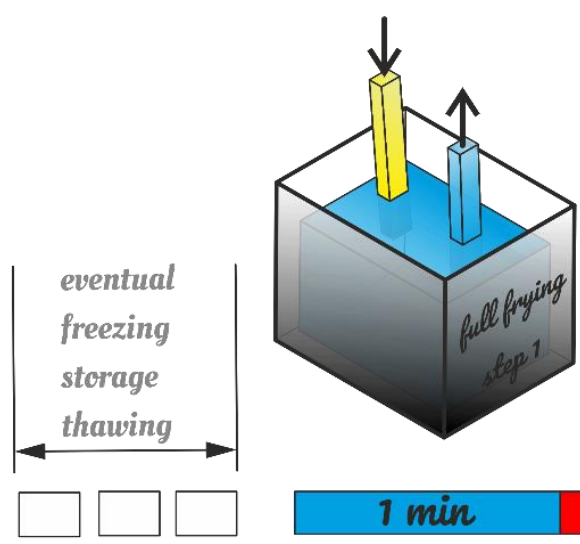

$1 \min$

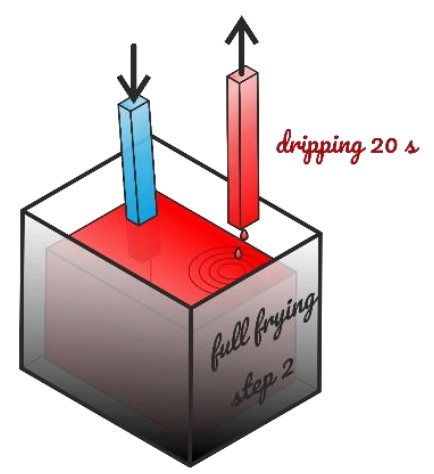

fime

Figure 2. Principle of oil labeling using dyed oil. Parfrying oil is not dyed.

Separation of surface and internal oil. Surface oil includes the oil film covering the product and oil inside surface cells cut during the preparation of the strips. During cooling, thermal contraction causes oil to be transferred from the top surface to the inner parts of the cells of the first layer (Achir et al., 2010). Surface oil was determined by immersing for $1 \mathrm{~s}$ each fried strip in $25 \mathrm{~mL}$ of petroleum ether at $40^{\circ} \mathrm{C}$. The subsequent steps are summarized in Figure 4.

Dissection of potato strips. The distributions of oil and residual water content in washed potato strips were reconstructed by dissection. The rubber core was mechanically separated from the glassy crust by using the difference of rigidity between both regions at room temperature. The somewhat artificial separation of the crust and of the core was interpreted by measuring the apparent glass transition temperature $\left(T_{g}\right)$ of the crust and of the crumb after dissection. It was verified that $T_{g}$ was globally above $30^{\circ} \mathrm{C}$ in the crust and below for the core. It corresponded to a critical water content of $0.27 \mathrm{~kg} \cdot \mathrm{kg}^{-1}$ of solid content and a critical temperature during frying of $105-106^{\circ} \mathrm{C}$ (see Figure 6 of Patsioura et al. (2015)). Similar $T_{g}$ value has been reported for gelatinized potato starch at a similar critical water content by Benczdi et al. (1998). The crust is therefore defined as the region of the material, which reaches a temperature equal or above 
$106^{\circ} \mathrm{C}$. It was additionally divided into three main regions: ends, edges and faces as shown in Figure $4 \mathrm{~b}$. They were indeed subjected to different drying and shrinkage rates.

Oil uptake in washed full fried strips. Oil uptake was determined by combining the samples from four fried strips. Samples were oven-dried at $103^{\circ} \mathrm{C}$ until constant weight before extracting oil with the Soxhlet reflux method. All recipients and tools were cleaned with the extraction solvent to avoid oil losses. Macroscopic water and oil contents were denoted $W_{S}$ and $F_{S}$, respectively, and expressed in a solid basis.

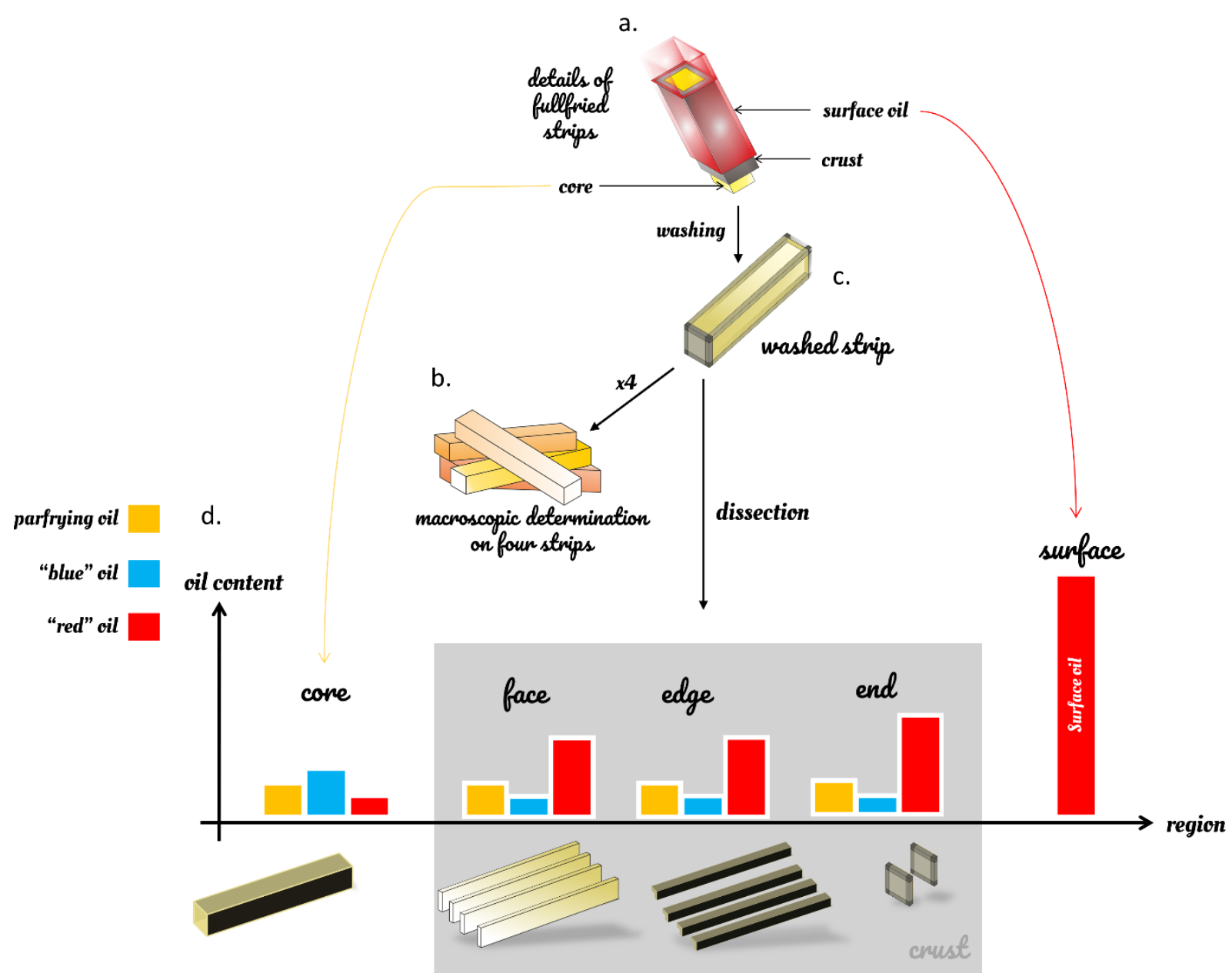

Figure 3. Principles of oil separation and dissection of full fried strips: (a) separation of adhering and penetrating oils; (b) macroscopic oil content determined from four washed strips; (c) details of the four dissection regions (core and three crusts regions: ends, edges, and faces); (d) decomposition of the different sources of oil at the scale of each region. 
Oil uptake determinations in the four dissected regions. Oil uptake inside each region was quantified on microsamples of ten milligrams by differential scanning calorimetry (DSC), as described by Aguilera and Gloria (1997). All measurements were duplicated, and the final results were averaged over three washed strips. The oil content was determined in wet microsamples by comparing the exothermic crystallization heat of the unknown sample with the specific heat of crystallization of the pure oil. All measurements were carried out on a DSC apparatus (model DSC1, Mettler Toledo, USA) equipped with an autosampler. Thermograms were acquired during a temperature cycle from $10^{\circ} \mathrm{C}$ down to $-60^{\circ} \mathrm{C}$ with a cooling rate of $1^{\circ} \mathrm{C} / \mathrm{min}$ and back up to $10^{\circ} \mathrm{C}$ with a heating rate of $1{ }^{\circ} \mathrm{C} / \mathrm{min}$. The typical thermograms of pure oil and the sample are shown in Figure 4. The cycle enabled us to verify that the exothermic peak of freezable water (peak numbered $\alpha$ ) did not overlap the crystallization exotherm of oil (peak numbered $\beta$ ). It is worth noticing that drying samples before DSC determinations would have oxidized oil and shifted its crystallization temperature. The solid content in each pan was verified after heating by convective drying at $110^{\circ} \mathrm{C}$. Prior drying, three holes were drilled in the cap of the pan. 


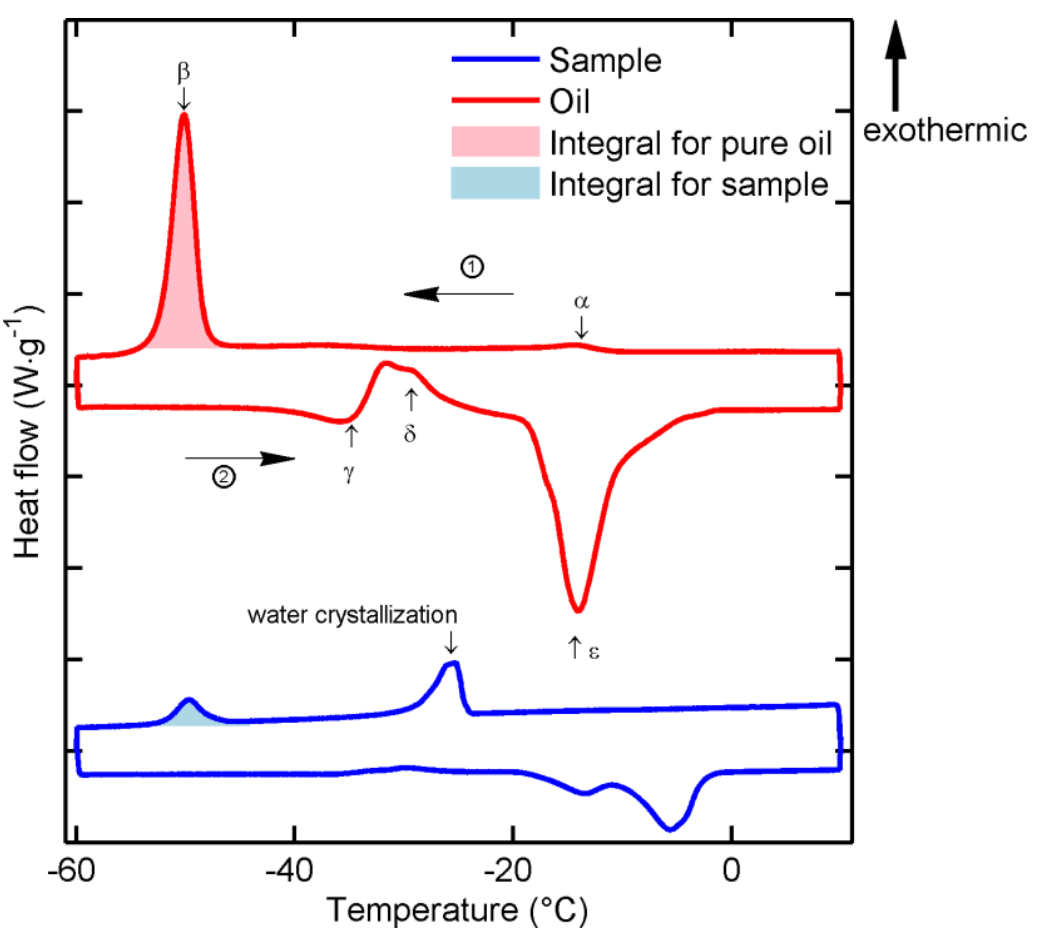

Figure 4: Example of thermograms of pure fried oil SO (red) and the French fry crust imbibed with the same oil (blue)

Separation and quantification of dyed oils. As the chosen dyes do not interact specifically with starch and cell walls constituents, the relative contents of "blue" and "red" oils were assumed to be proportional to the concentration of each dye. The concentrations in SR (Sudan Red) and SB (Sudan Blue) in the oil bath and in the sample were determined by deconvolving their ultraviolet-visible spectra in mixtures (i.e., without any physical separation). Since the spectra of SB and SR are overlapping and are present at very different concentrations in samples, the non-negative least-square deconvolution procedure of Gillet et al. (2011) was applied. The methodology is briefly summarized hereafter and illustrated in Figure 5. Spectra of aliquots of the unknown mixture were collected for different dilution ratios $(D)$ in neat oil (see Figure 5a). A deconvolution procedure was applied for each value of $D$, and the slope of the deconvoled spectrum versus $D$ was estimated for the linear domain of correlation (see Figure $5 \mathrm{~b}$ ). The slope estimated the oil concentration after calibration with binary mixtures (neat oil+dye). The dilution procedure enabled to zoom on important regions of the spectra while avoiding signal 
saturation. Specific challenge tests with ternary mixtures (neat oil+SR+SB) were carried out to verify the absence of bias (each dye is not confused by the other) and to estimate the experimental error below $2 \%$.
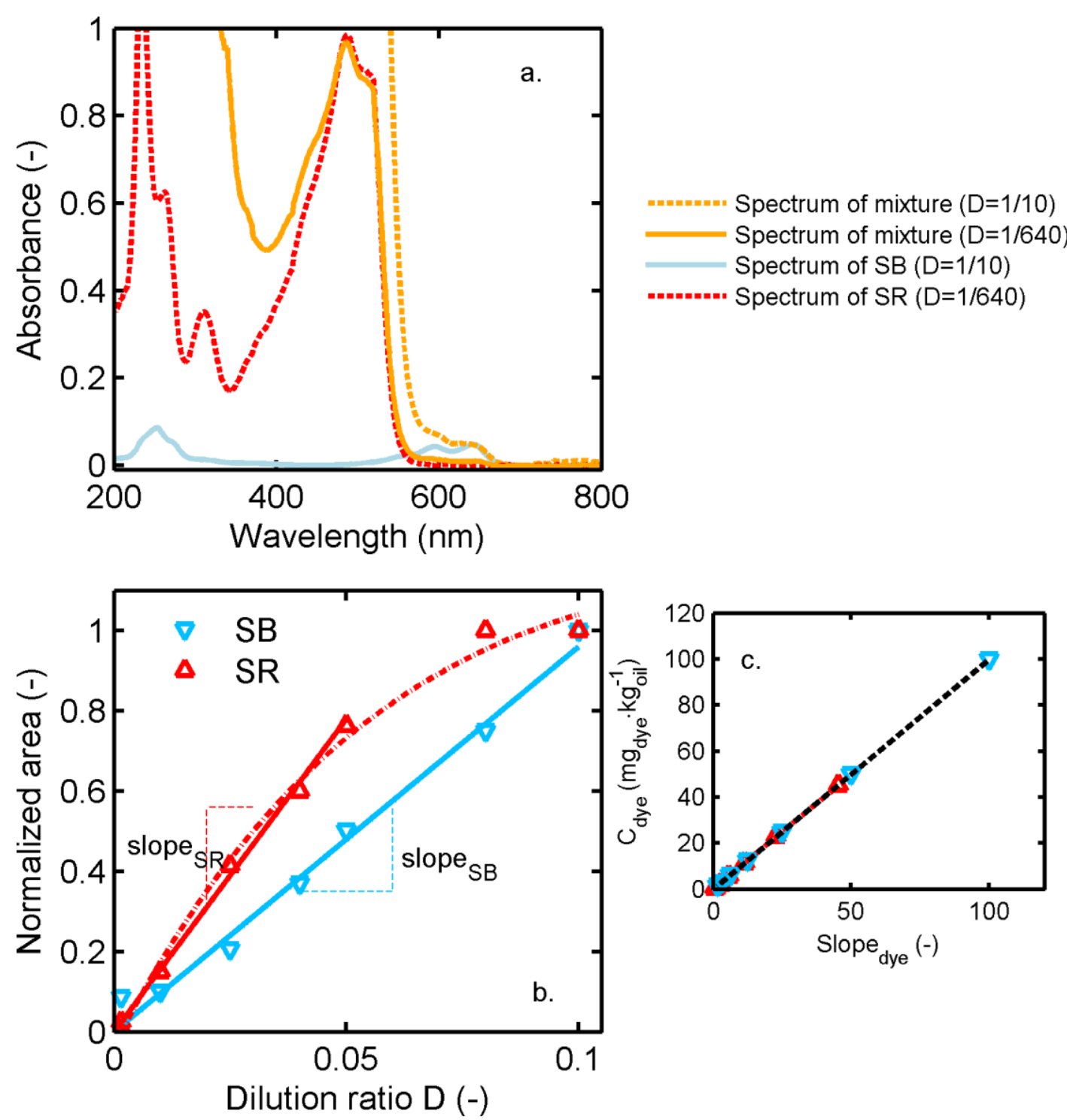

Figure 5. Principles of the separation of oil contents dyed with Sudan Blue (SB) and Sudan (Red): (a) UV-VIS spectra of SB and SR mixture and pure compounds in oil; (b) linear relationship between the intensity of spectra and dilution ratio; (c) calibration curves in sunflower oil. 


\subsection{Microscopic observations of oil distribution and microstructure in full-fried strips}

Visible imaging. Cross-sections were immediately imaged after finish frying using a digital microscope (model Mighty Scope 5.0M, Aven, MI, USA). The auto-controlled lighting source (ring of 6 white LEDs) ensured a uniform and constant illumination between samples. Segmentation of regions with blue and red oil colors was achieved by K-mean clustering of the images in the CMYK space.

Micro-computed X-ray tomography. The microstructure of full-fried potato strips were observed non-invasively in micro-computed X-ray tomography (model V |tome| X combined with DatosX_Rec reconstruction software, General Electrics, USA) at a resolution of $1200 \mathrm{x}$ 1200 x 1000 voxels. Oil and solid contents were segmented using the watershed transform.

\subsection{Online measurements during finish frying}

Temperature measurements and temperature field reconstruction. Temperatures inside parfried strips were recorded at five different positions at a frequency of $3 \mathrm{~Hz}$ with five $0.5 \mathrm{~mm}$ thick cylindrical thermocouples (type T, TC SA, France) connected to a data acquisition system (NI 9214 module mounted on a NI cDAQ-9178 chassis, National Instruments, USA). An attempt of reconstruction of the 2D temperature field (in the sagittal plane) was proposed by fitting the 2D mechanistic simulation detailed by Vitrac et al. (2000) to temperature measurements.

Steam bubbling imaging. Bubbles escaping from a single strip were imaged using a modified deep fryer (model Semi-Pro, 4L, SEB, France) equipped with three windows below the surface. The strip was placed horizontally ca. $80 \mathrm{~mm}$ between the two parallel windows and illuminated at a right angle through the third window with non-focused light emerging from an optical fiber with a diameter of $10 \mathrm{~mm}$. Images (resolution $1920 \times 1200$ ) were acquired at $\sim 160$ frames per 
second using a CMOS digital camera (GS3-U3-23S6M-C, Point Grey, Canada) equipped with a close focus zoom (10X, 13-130 mm, FL Edmund Optics, UK).

\subsection{Evidencing longitudinal oil flow at the surface and inside strips}

In the conventional description of oil uptake, oil penetrates inside the product by following the shortest route connecting the external surface to internal regions. The capacity of oil to flow internally over large distances along the strip was tested in a special configuration shown in Figure 6. A parfried strip initially frozen with a surface temperature of $-13^{\circ} \mathrm{C}$ (verified with an infrared camera, model Ti9, Fluke, USA) was half immersed in blue-dyed oil at $180^{\circ} \mathrm{C}$ while the other half was exposed to the ambient temperature $\left(\sim 30^{\circ} \mathrm{C}\right)$. The configuration exacerbated the possibility of steam to flow from the immersed vaporization region (sources below the immersion lines) to upper regions (sinks above the immersion line) where it could condensate. The surface displacement of oil by capillarity was imaged at $\sim 160$ FPS for $40 \mathrm{~s}$. The averaged ascensional height of the oil front (along the vertical direction $z$ ), $H(t)$, was calculated as

$$
H(t)=\frac{\iint I_{\text {Cyan }}(t, x, z) z d x d z}{\iint I_{\text {Cyan }}(t, x, z) d x d z}
$$

where $I_{\text {Cyan }}(t, x, z)$ is the intensity of the Cyan component at positions $x$ and $z$ in the image. The height $H(t)$ was used as the reference position attainable spontaneously by oil, that is by displacing air in the crust, and was compared with the position of the oil front inside the product. After different attempts of imaging online the displacement of the oil inside the strip by analyzing transmitted light, dissection was preferred due to the large varieties of internal patterns and the heterogeneities of the dynamics. The cut was initiated from the non-dyed top to prevent the dye from spreading in the frozen structure. 

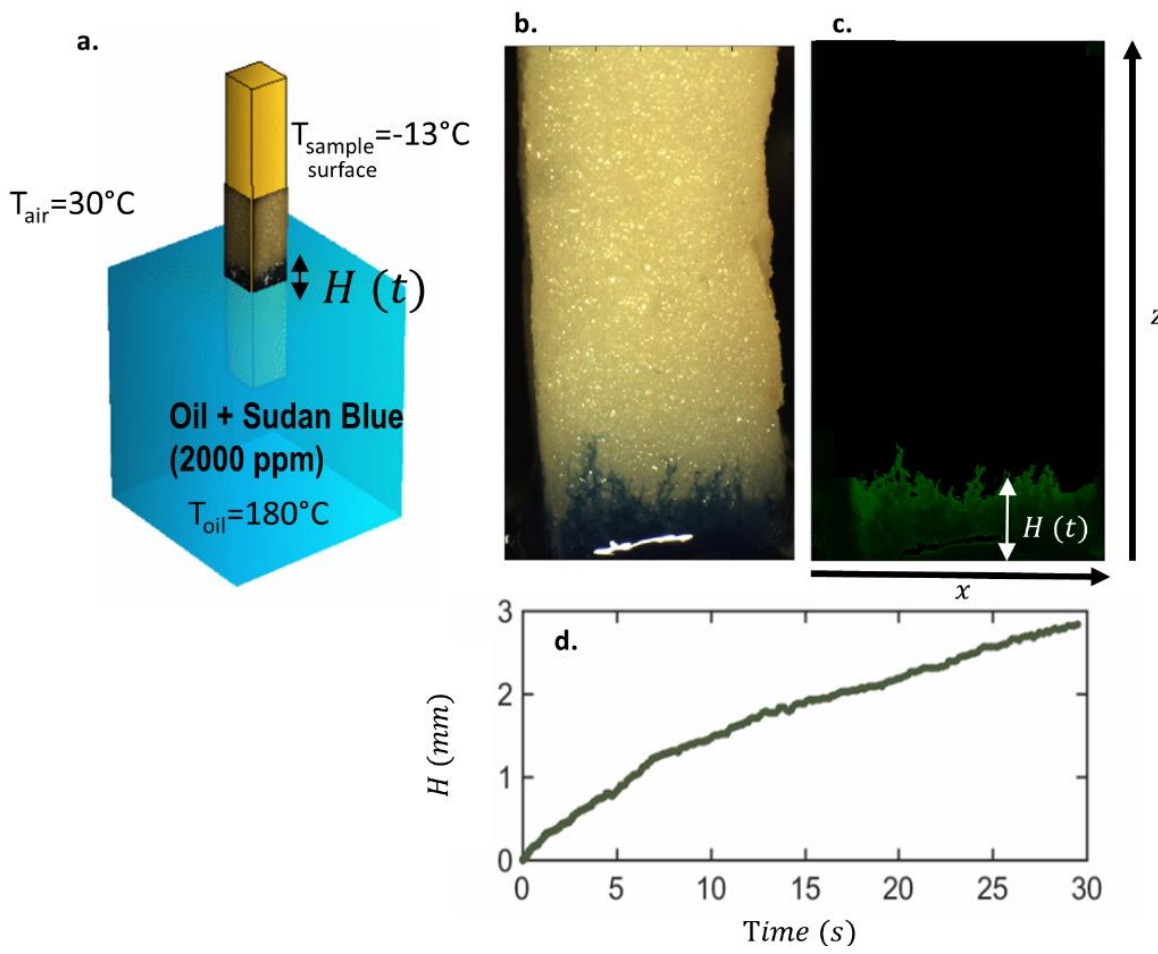

Figure 6. Experimental configuration to study the vertical displacement of oil in an initially parfried frozen Frenchdry: (a) region observed, (b) typical observation, (c) same image after segmentation, (d) average oil position with time (calculated from Eq. (1)).

\section{Results and discussion}

In this study, oil uptake is analyzed from two independent perspectives: i) as an entropydriven phenomenon occurring at cellular scale when the product is removed from the oil bath and ii) as the consequence of large-scale fluctuations of the pressure field during the immersion stage. The pioneer observations of the two oil populations have been reported in parfried frozen products, but not evidenced in similar products prepared from fresh potatoes (Patsioura et al., 2016). The sufficient condition enabling oil penetration during immersion is not known. It has been already noticed that oil was penetrating only during the first minute and not beyond (Patsioura et al., 2016). The next section analyzes systematically the combinations of different treatments and initial states on the different populations of oil: oil absorbed during the first 
minute of frying (dyed in blue and so-called "blue" oil) and oil absorbed once the product is removed from the oil bath (dyed in red and so-called "red" oil).

\subsection{Occurrence and distribution of "blue" oil in fried products}

Effect of combined pretreatments. Seven combinations of pretreatments were considered, labeled from A to $\mathrm{G}$, and compared between laboratory and industrial (letter with *) processing conditions. Forensic observations of forty-one French-fries sectioned longitudinally are shown in Figure 7. All samples were subjected to the same finish-frying treatment comprising $1 \mathrm{~min}$ in blue oil and $4 \mathrm{~min}$ in red oil at $180^{\circ} \mathrm{C}$. The depicted results show a small fraction of all collected observations, and the repetitions presented row-wise (from 1 to 6 ) were chosen to be representative of the different oil imbibition patterns.Oil distribution and their origin assignation (blue or red) were set after image analysis. 


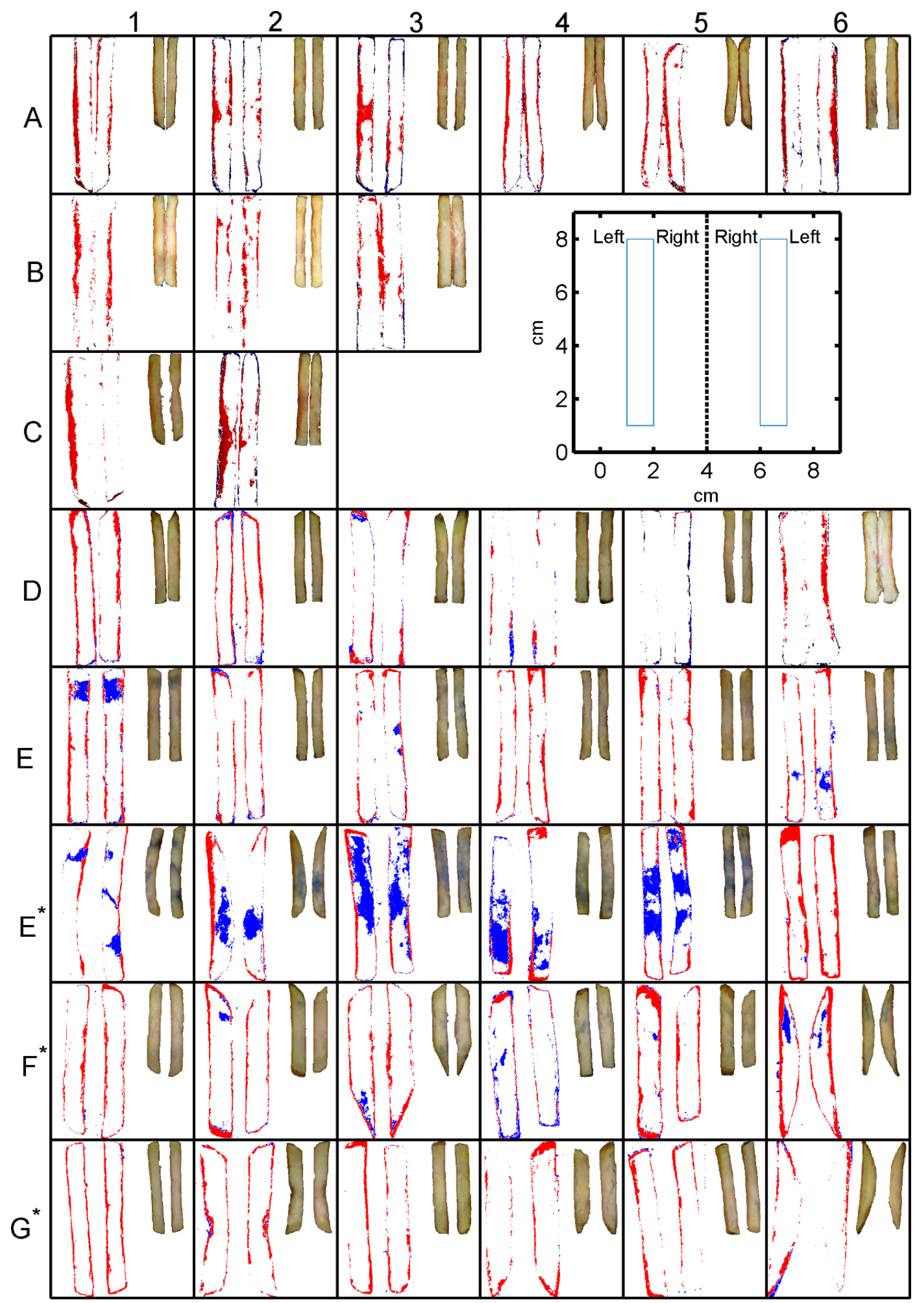

Figure 7. Observations of the longitudinal cross sections of potato strips after the treatments listed in Figure 1 (vertically) and their repetitions (horizontally). For each observation, segmented images are shown on the left (artificial colors: blue=blue oil, red=red oil) and the original images on the top right (scale 1:2). 
Red oil covered all samples in a quite similar manner. Regions filled with larger red spots were associated with artifacts produced during sectioning. Red oil was located almost exclusively in the crust with thicknesses varying from 0.5 to $2 \mathrm{~mm}$. Blue oil appeared significantly only in samples $\mathrm{E}, \mathrm{E}^{*}$ and $\mathrm{F}^{*}$. These samples shared three steps: parfrying, freezing, and storage at frozen state. Noticeably, ice was present in the three types of samples before finish-frying. Removing any of the previous steps or performing a full thawing before finish frying prevented the apparition of blue oil. Oil pickup during frying (blue oil) was maximum in industrial products $\left(\mathrm{E}^{*}\right)$. The differences between $\mathrm{E}$ and $\mathrm{E}^{*}$ behaviors were associated with longer storage times in industrial products (from several weeks to several months). It was verified that a partial thawing reduced oil absorption during the immersion stage.

In the remaining part of the study, only the mass transfer in the samples $\mathrm{E}^{*}$ will be reported. Internal migration of blue oil was observed more frequently and more symmetrically between both ends on such industrially parfried products. Similar trends were observed when similar products were prepared in the laboratory, but with more variability due to shorter storage time at frozen state. As a result, it was hinted that similar phenomena were taking place in both situations and that no specific phenomena were associated with the industrialization of parfrying and storage steps. Additionally, the patterns of red oil adhesion and penetration are thought to be very similar between all the eight conditions (from A to $\mathrm{G}^{*}$ ).

Oil penetration pathways in samples $E^{*}$. In detail, blue oil was never distributed homogeneously, symmetrically, or followed the contours of the strips in samples $\mathrm{E}^{*}$. The penetration depth, its occurrence inside the same product, and between products looked random. Some general rules could be, nevertheless, drawn. Blue oil could reach the center, cross the symmetry plane, and could penetrate through a few numbers of entry points. Penetration angles 
were also random with several oblique directions. Due to the absence of penetration from ends, oil looked penetrating from equatorial regions towards ends. Penetration pathways of blue oil corresponding to $\mathrm{E}_{1} *$ are analyzed with higher magnification in Figure 8 with details at cellular scale in Figure 9.

a.

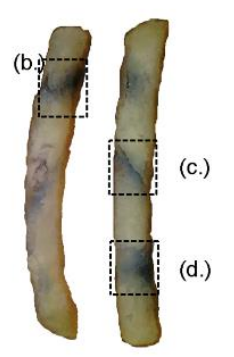

b.

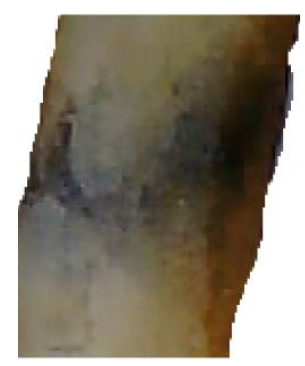

c.

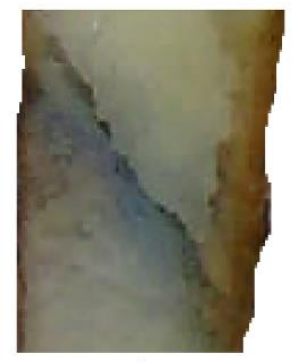

d.

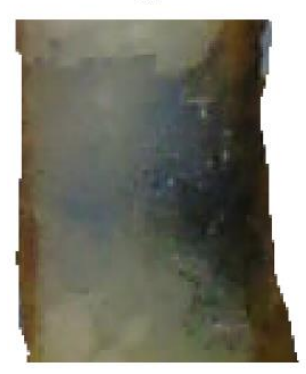

e.

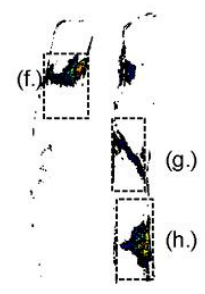

f.

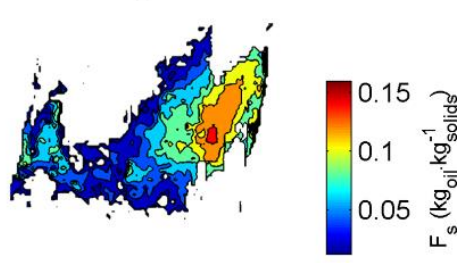

g.

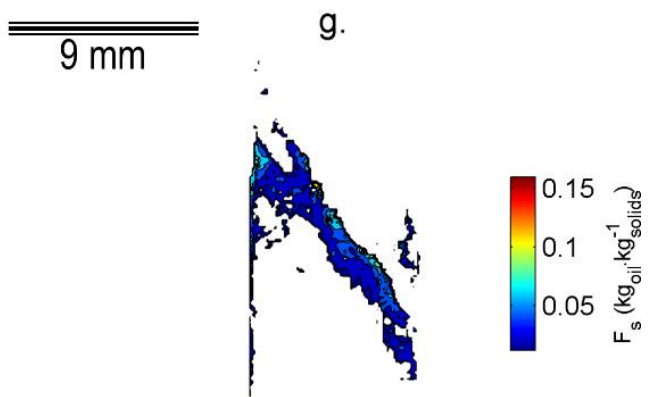

h.

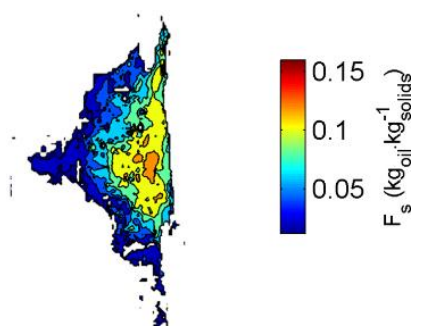

Figure 8. Details on penetration pathways of blue oil in the sample $E_{1}^{*}$ shown in Figure 7 (a-d). Concentrations of oil are shown as isocontours in e-f. 
Blue oil penetrated inside the same product through three defects and fractures. For each spot, the gradient of blue intensity suggested the likely direction of oil transport. Blue oil from spots in Figure 8b,d c from large defects in the crust located right- and the left-hand-side, respectively. Blue oil also imbibed the wet core far below the crust until reaching the opposite side. The spot in Figure 8c was expanded from the left and spread subsequently along an oblique fracture. Dispersion in the wet core perpendicular to the main direction was also observed. Microscopic observations of Figure 9 demonstrate that blue oil infiltrated the gaps between parenchyma cells deep inside the product, regardless of the distribution of water inside the product.

a.

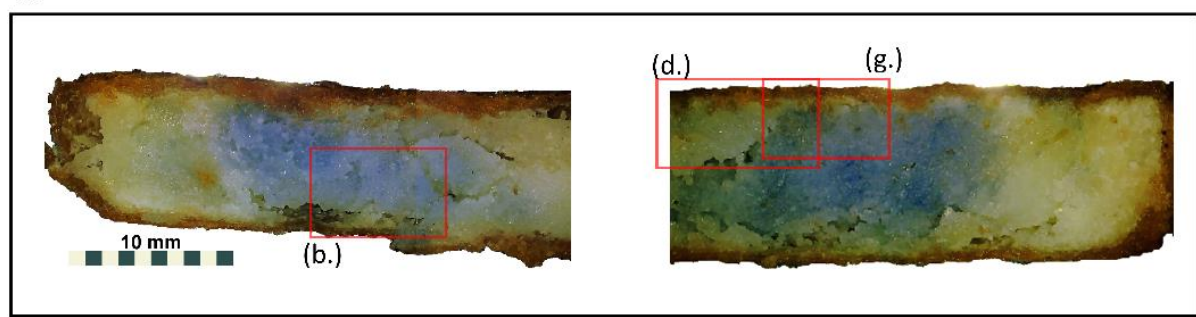

b.

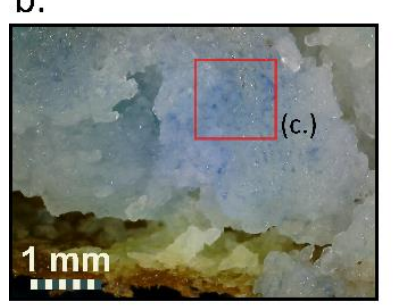

d.
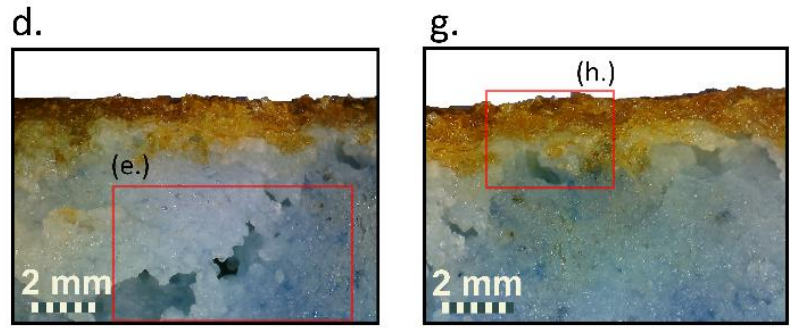

c.

e.
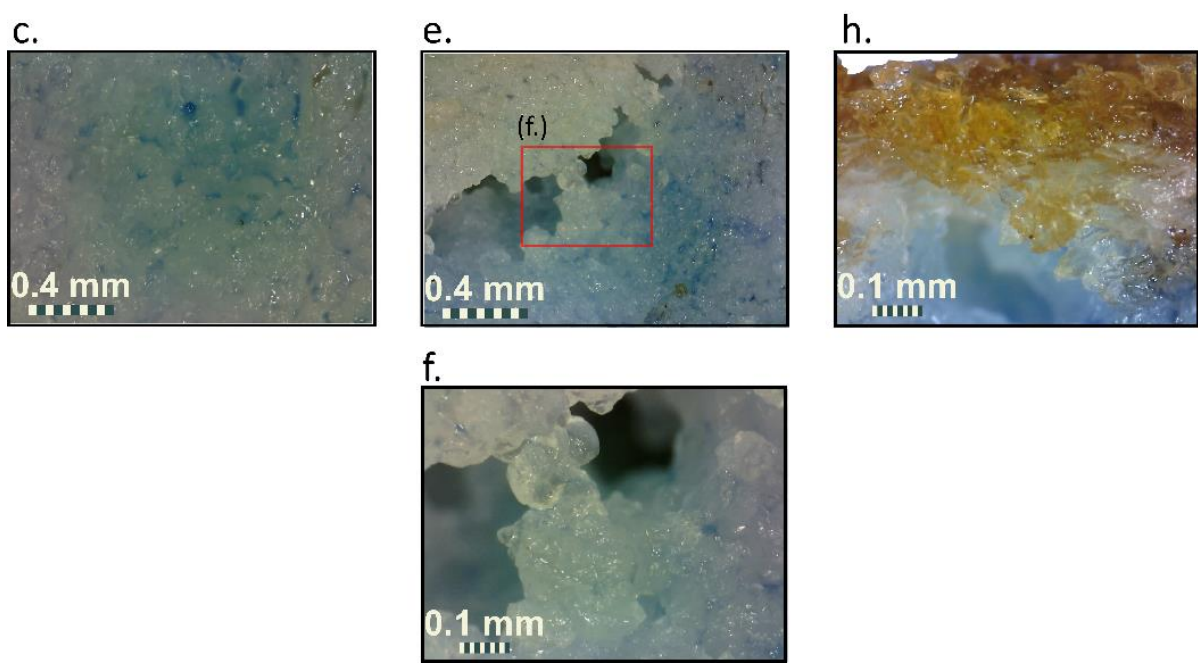

Figure 9. Microscopic observations at different scales of the distribution blue oil in a typical French-fry $\left(E^{*}\right)$. 
The dispersion of oil in water-rich regions was surprising at first sight, but it was made possible by the loss of cohesion of middle lamella separating cells during freezing. Parenchyma cells tended to separate into small "bags" during frying while being turgid by the presence of swollen starch. As a result, it was the combination of treatments: gelatinization of starch during parfrying and tissue injuries during storage at frozen state, which enabled the penetration of oil from large cracks open to the outside to the wet inner core. This new description contrasts dramatically from the oil percolation process between intact cells (Patsioura et al., 2015), which proceeds at a very slow pace due to the passage of oil through small defects.

Oil mass balance. The conventional description of oil uptake assumes that oil fills partly the voids left by water (Vitrac et al., 2002). Dry regions are thought to be richer in oil than the ones filled with water Corollary. Water and oil mass balance depicted in Figure 10 showed a more complex picture in parfried frozen products. 

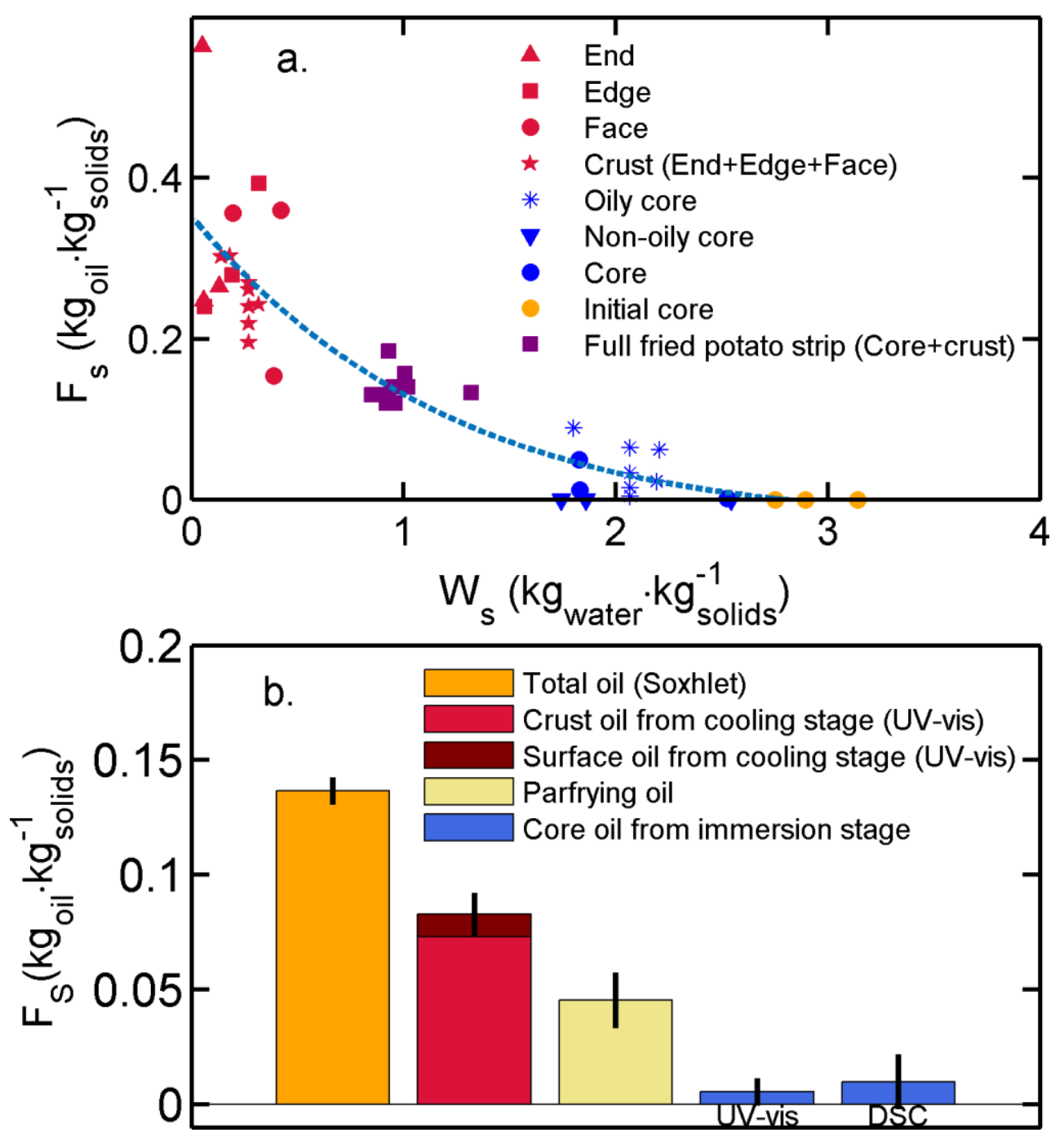

Figure 10. Distribution of oil in full-fried strips (a) between the four different dissected regions and (b) according to its origin. The dashed line in (a) plots the apparent correlation between local determinations of $F_{S}$ and $W_{S}$. The black lines in (b) plot the standard deviations.

Oil is more uniformly distributed inside the sample with higher concentrations in edges and faces. The residual parfrying oil was obtained by difference (not blue, not red); it represented approximatively one-third of final oil. The comparison between the initially parfried and fullfried strip showed that approximatively half of the initially present parfrying oil is lost during finish frying and replaced by red oil. Blue oil was exclusively present in wet regions and represented no more than $5 \%$ of the total oil, with significant variations between samples. As oil surface represents $6 \%$ of the total oil, oil in parfried frozen products is a surface phenomenon controlled by surface-to-volume ratios. The average oil content in French-fries does not 
correspond with the oil content of any region and appears in the tested conditions appeared as the middle of high (red) and low (blue) content values. The trend line shown in Figure 10a is very indicative and confirmed the large variations around the averaged content in the crust appeared for red oil and also existed for blue oil in the core. As a rule of thumb, regions that dryed faster, such as ends and edges, exhibited a higher replacement ratio of water by oil. The likely reason would be the higher damages due to the bidimensional shrinkage occurring near edges and corners.

\subsection{Reconstruction of 3D networks followed by blue oil}

The heterogeneous patterns of blue oil distribution in parfried frozen French-fries does not originate from the organization of the potato tissue and should be analyzed in relationship with the macroscopic damages in the product. Three common cellular damages in finished products are shown in Figure 11. Damages appear as oblique fractures in cross-section planes, sometimes branched when the damage levels are high. As the same patterns appear in distant crosssections, they should be envisioned as "extruded shapes" propagating along with the sample. 
a.

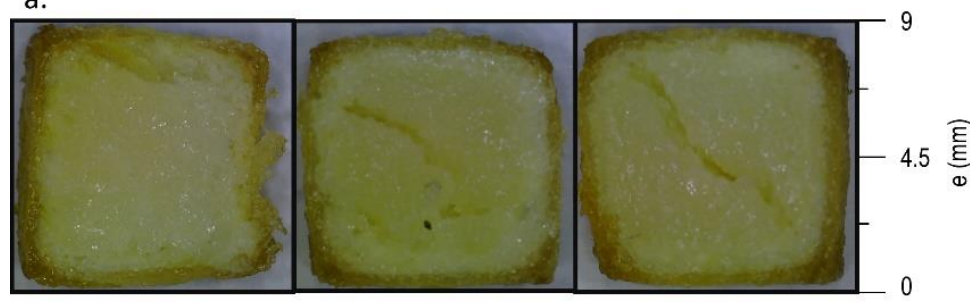

b.
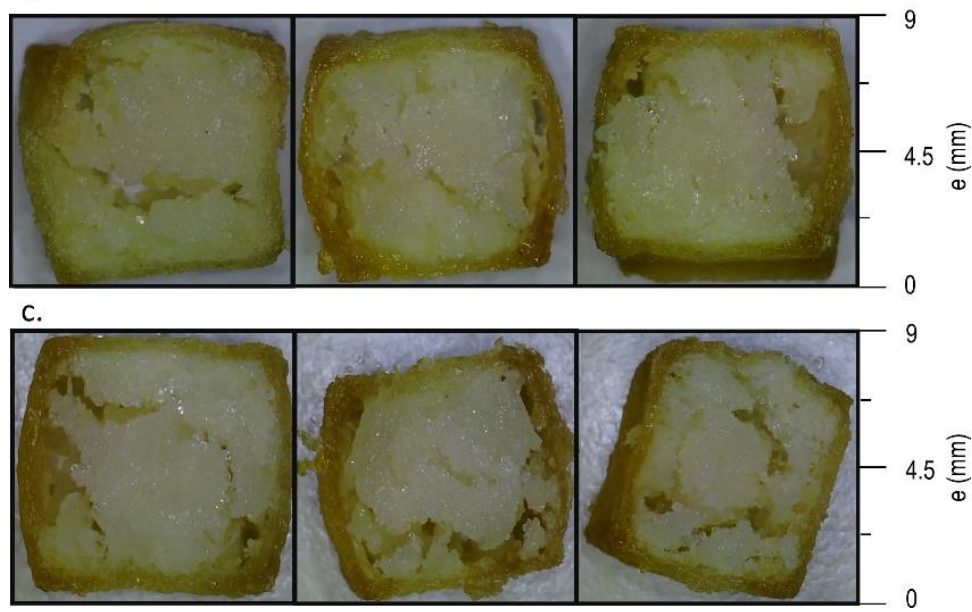

Figure 11. Visible observations of tissue damages in three samples in three $\mathrm{E}^{*}$ samples (vertically) at three different positions (horizontally) corresponding to $2 \mathrm{~cm}, 4 \mathrm{~cm}$ and $6 \mathrm{~cm}$ from the end: samples with (a) low, (b) intermediate and (c) high damage levels. 


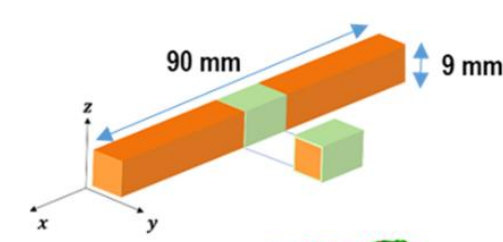

a)

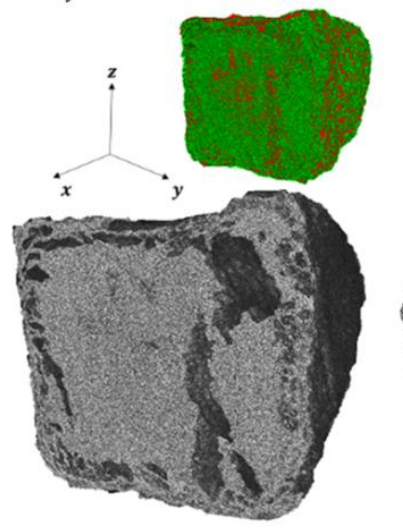

b)

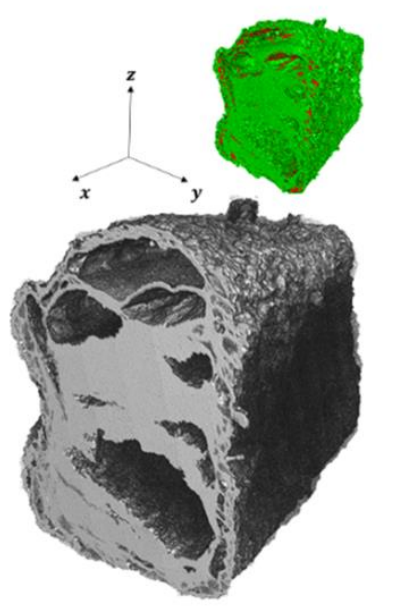

c)

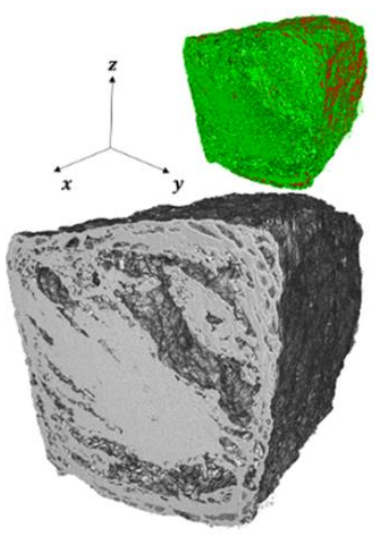

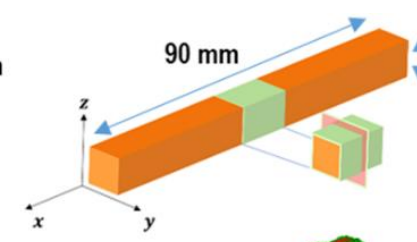
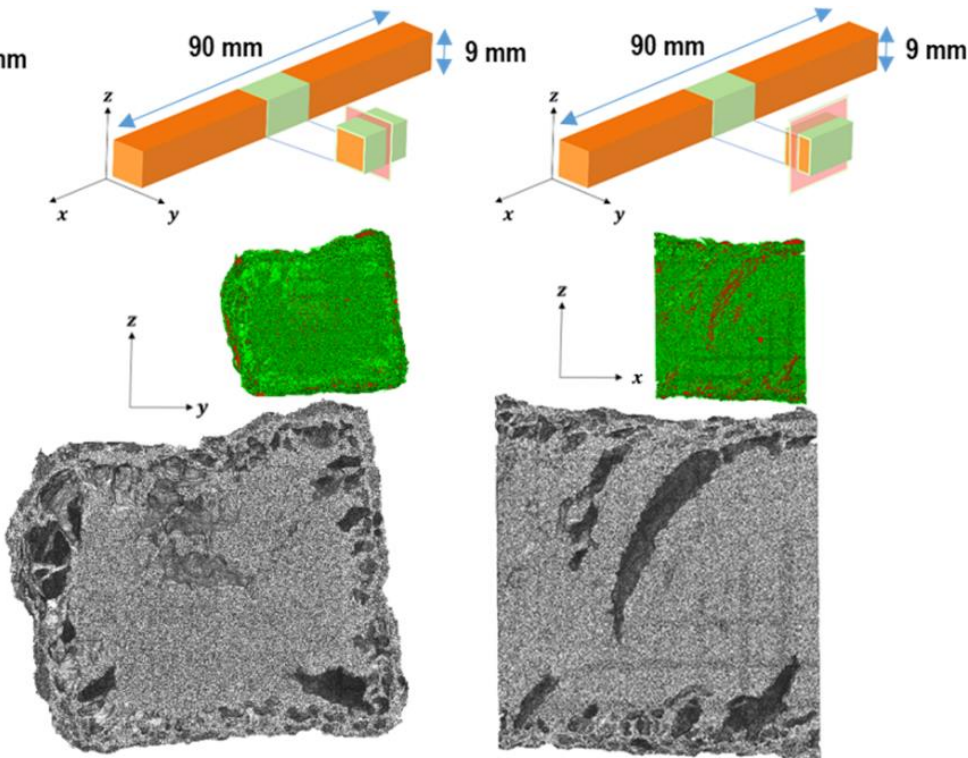

$x$
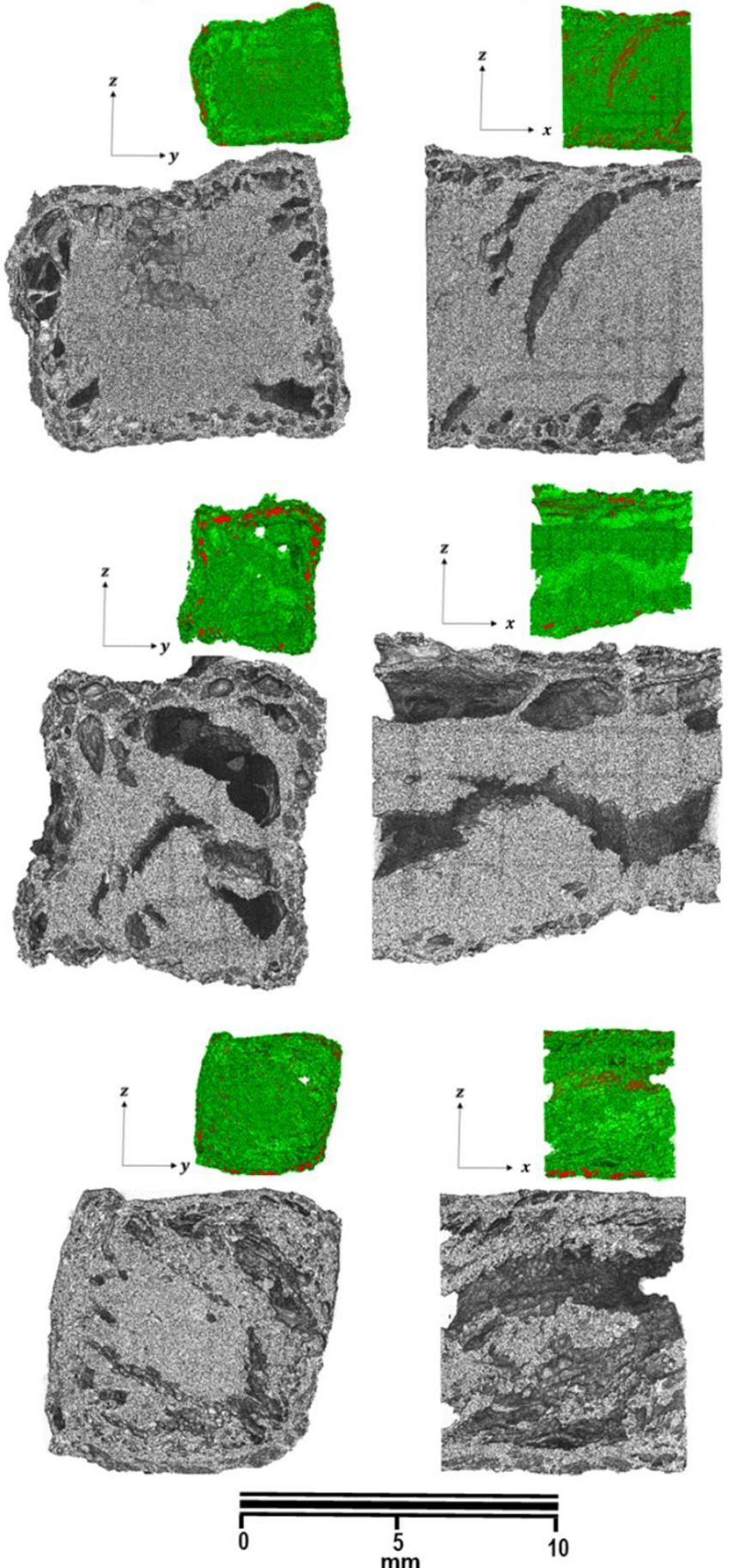

Figure 12. Computerized microtomography of defects in parfried frozen French fries after finish frying in three different samples $\mathbf{E}^{*}$ (denoted a-c). Phase reconstructions are proposed in false colors showing cell walls and starch content in green, and oil in red. 
The 3D-connectivity of damages (high damage levels) was imaged by X-ray tomography. Oil menisci inside the product were identified qualitatively by segmentation. Typical structures are depicted in Figure 12. They demonstrate that the fractures are larger in the longitudinal direction with lengths reaching several centimeters. They cannot be guessed from the outside of French fries. They avoid the corners where the cohesion between the crust and the core is stronger; they develop in wet and rubber regions where the initial pressure during finish frying enabled their expansion. Contrarily to most of the previous descriptions, the largest dimension of cavities does not appear aligned with the smallest cross-section but along the length of the French-fries instead. The maximum size of cavities can reach 2 centimeters or more.

\subsection{Driving forces of oil penetration during the immersion stage in parfried frozen French- fries}

The conditions during finish-frying of parfried frozen products differ from those met by unfrozen ones because water is present simultaneously in its three states (ice, liquid, steam). The vaporization front coexists with a moving melting front inside the product. Within an infinitely long French-fry, both fronts are expected to be concentric and separated by liquid water. In this ideal geometry, the presence of fissures parallel to the main faces is not expected to modify the separation of the frozen phase with the one filled with steam. This situation contrasts with their arrangements close to ends, where heat transfer is initially more intense (larger surface area). The fronts are expected to shift to conic shapes (i.e., focaloid) so that they intercept any fissure parallel to main faces. This configuration could induce a flow of steam towards icy regions. As ice and steam cannot exist in frying conditions, the internal pressure can be temporarily destabilized in the region of the fissure. These effects are expected to disappear as soon as the melting front reaches the center of the French-fry. 
Local temperatures. The typical kinetics of heating in a parfried frozen French-fry is shown in

Figure 13 for different repetitions. Due to the variability in the initial temperature, the delay in melting the ice fully inside the product varies from $20 \mathrm{~s}$ to $45 \mathrm{~s}$. The conical shape of the fronts is exemplified by the inequality $T_{4} \geq T_{5} \geq T_{6}$.

a.
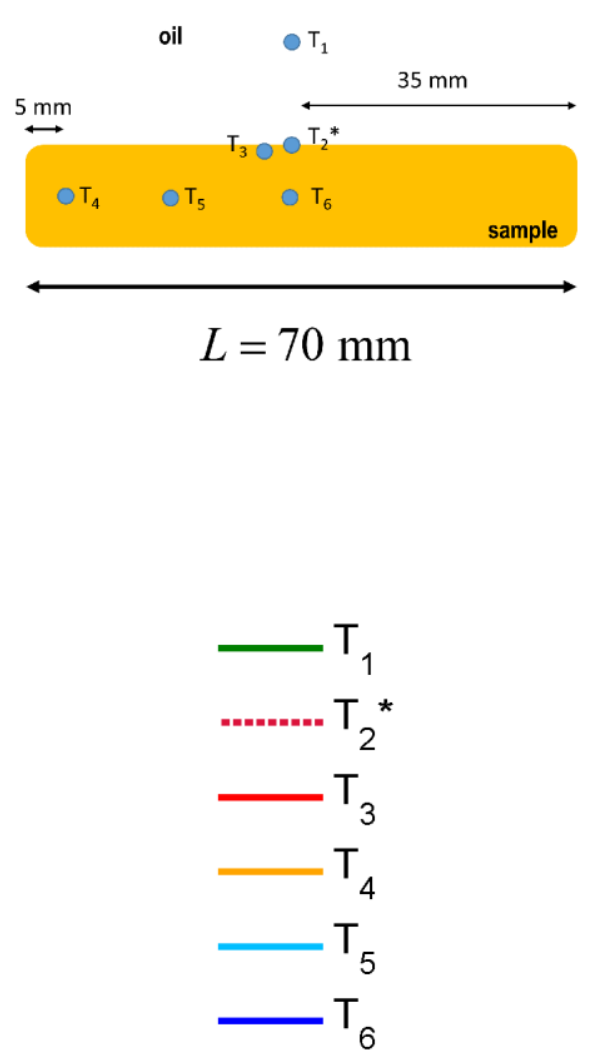

b.
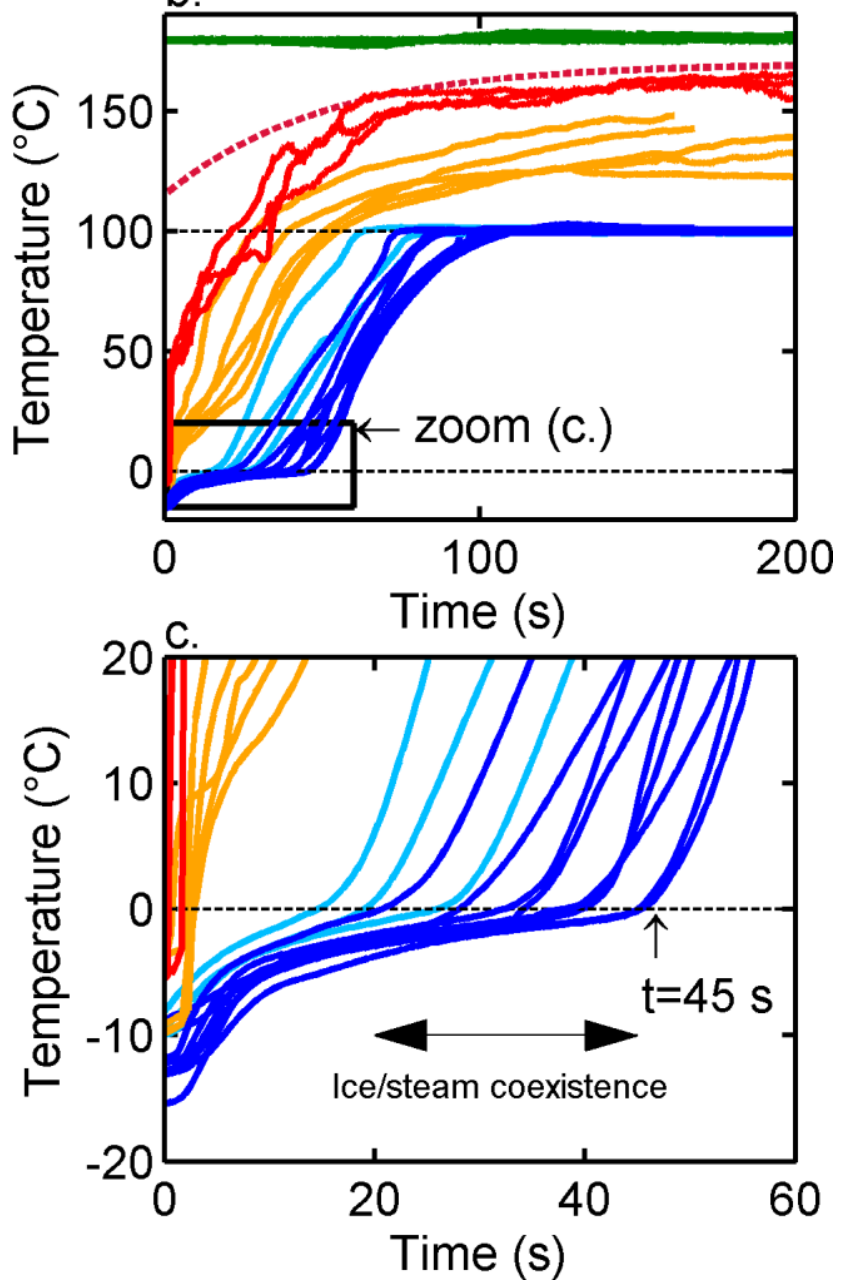

Figure 13. Local temperature measurements in parfried frozen French-fries $\left(E^{*}\right)$ during finish-frying at $180^{\circ} \mathrm{C}$. (a) thermocouple locations; (b) kinetics including up to 10 repetitions; (c) details during the first minute. T2* is the extreme surface measured by Ref.(Hubbard and Farkas, 2000).

Steam bubbles are observed almost immediately after immersion, but the boiling condition inside the crust (position of thermocouple $T_{3}$ ca. $1 \mathrm{~mm}$ below the surface) occurs only $20 \mathrm{~s}$. As a result, the possibility of coexistence of steam and ice inside the product is thought to last from 0 to $25 \mathrm{~s}$. Because this moment is volatile, it could explain the variability in blue oil observed 
in parfried frozen samples E and $\mathrm{E}^{*}$ (see Figure 7). In partly thawed products (samples $\mathrm{F}^{*}$ ), the time window enabling the coexistence of ice and steam is even shorter, and the amount of blue oil is, therefore, lower and more variable (see Figure 7). In fully unfrozen products (samples $\mathrm{G}^{*}$ ), no blue oil is detected although the products were initially parfried and stored frozen. The presence of fissures alone is not sufficient to trigger the penetration of oil during finish frying.

Reconstruction of the longitudinal steam flow inside the product during the immersion stage.

The internal pressure raises above the external pressure, $P_{0}$, when the displacement of vaporization leave behind it a rigid and hygroscopic crust opposing to subsequent heat and mass transfer (Vitrac et al., 2000). Longitudinal heterogeneities in the pressure field beneath the crust are suspected to be capable of reorienting the flow of steam along fissures and cracks from one end towards the core. A sufficient condition is that the fissure is partly filled with ice where steam can condensate, as illustrated in Figure 14a. An attempt of reconstruction of the pressure field along the direction $x$ is proposed in Figure 14b-d by recording the flow of steam bubbles in the vertical plane $(x, z)$ during the first minute of immersion of a parfried frozen strip (type E) at a rate of 160 frames per second. The intensity of the light deviated by bubbles was averaged over approximately $2 \mathrm{~s}$ to estimate the longitudinal density of the vaporization rate, $\langle q(x)\rangle$, with SI units in $\mathrm{kg} \cdot \mathrm{m}^{-2} \cdot \mathrm{s}^{-1}$. Due to the higher exchange surface area close to ends, the vaporization rate is initially higher close to extremities. Indicative temperature contours were reconstructed from measurements of Figure 13 and simulations (Vitrac et al., 2000). In the crust, the pressure is expected to drop along the shortest distance to reach the external surface, denoted $l(x)$, so that it is connected to the bubbling density (SI units $m \cdot s^{-1}$ ) as: 


$$
\langle q(x, t)\rangle=\frac{K_{\text {crust }}}{\mu_{v}} \frac{P(x)-P_{0}}{l(x)}
$$

within the intrinsic permeability through the crust (SI units $m^{2}$ ), assumed to be uniform and isotropic (Ziaiifar et al., 2008), and $\mu_{v}$ the dynamic viscosity of steam.

A flow of steam along one fissure connecting the crust with the frozen core may occur when the pressure gradient along $x$ is favorable. The flow rate reads along the hypothetical fissure:

$$
Q_{v}^{\text {fissure }}(x, t)=-A_{\text {fissure }} \frac{k_{v} K_{\text {fissure }}}{\mu_{v}} \frac{\partial P(t, x)}{\partial x}=-A_{\text {fissure }} k_{v} \frac{K_{\text {fissure }}}{K_{\text {crust }}} \frac{\partial}{\partial x}\langle q(x, t)\rangle l(x, t)
$$

where $A_{\text {fissure }}$ is the surface area, $K_{\text {fissure }}$ the intrinsic permeability of the fissure and $k_{v}$ the relative permeability to steam. The amount of accumulated steam $m_{v}^{\text {fissure }}$ in the fissure below the vaporization region (i.e., no generation of steam in the fissure) is given by the mass balance:

$$
\frac{\partial m_{v}^{\text {fissure }}}{\partial t}+\frac{\partial\left(\rho_{v}^{(T)} Q_{v}\right)}{\partial x}=0
$$

with $\rho_{v}^{(T)}$ the density of superheated steam.

According to Eq. (3), water vapor flows longitudinally as a consequence of the faster thickening and higher bubbling rate in corners and edges. Dense crusts filled with gelatinized starch may consequently increase pressure drop and enhance the lateral circulation of steam. The geometry characteristics (orientation, length, section) and the intrinsic permeability of the crumb control the distance enabling the transport of water vapor. Such a transport does not require substantial deviation to local thermodynamical equilibrium, but only a partly saturated medium, where water vapor can coexist with its liquid counterpart. The coexistence is likely under high heat fluxes when the cell walls are enough damaged to enable the expansion of steam in free voids. 
The examples of reconstruction shown in Figure 14 are based on a crust thickness determined by the distance between the isocontours $T=T_{\text {sat }}=102^{\circ} \mathrm{C}$ and the surface. Eq. (4) was integrated with an upwind scheme over a short period of five seconds. The conic shape of the end of the drying front confirms that a net transport from the right (end) to left (central region) is possible and even very likely during the first $30 \mathrm{~s}$. Beyond this period, the residual frozen region appears too far from ends to enable the condensation of steam. From this description, the driving force associated with the penetration of oil during immersion in deep regions filled with liquid water should be envisioned as a thermodynamic process. The work associated with the condensation of steam is converted effectively in pressure-volume work, enabling oil to penetrate "in force" within extracellular space. This mechanical work is produced by the longitudinal (or oblique) displacement of the steam flow from a hygroscopic region towards the frozen core. This transfer requires both fissures or defects and an initial frozen state. It happens mainly when a random fissure connects the vaporization and thawing fronts. As both fronts deepen rapidly, the phenomenon is highly elusive and random. The elastic behavior of the crumb should contribute to sustaining the expansion of fissures and the propagation of dislocation over large period of time (10-30 s) and distances (several centimeters). 
a.
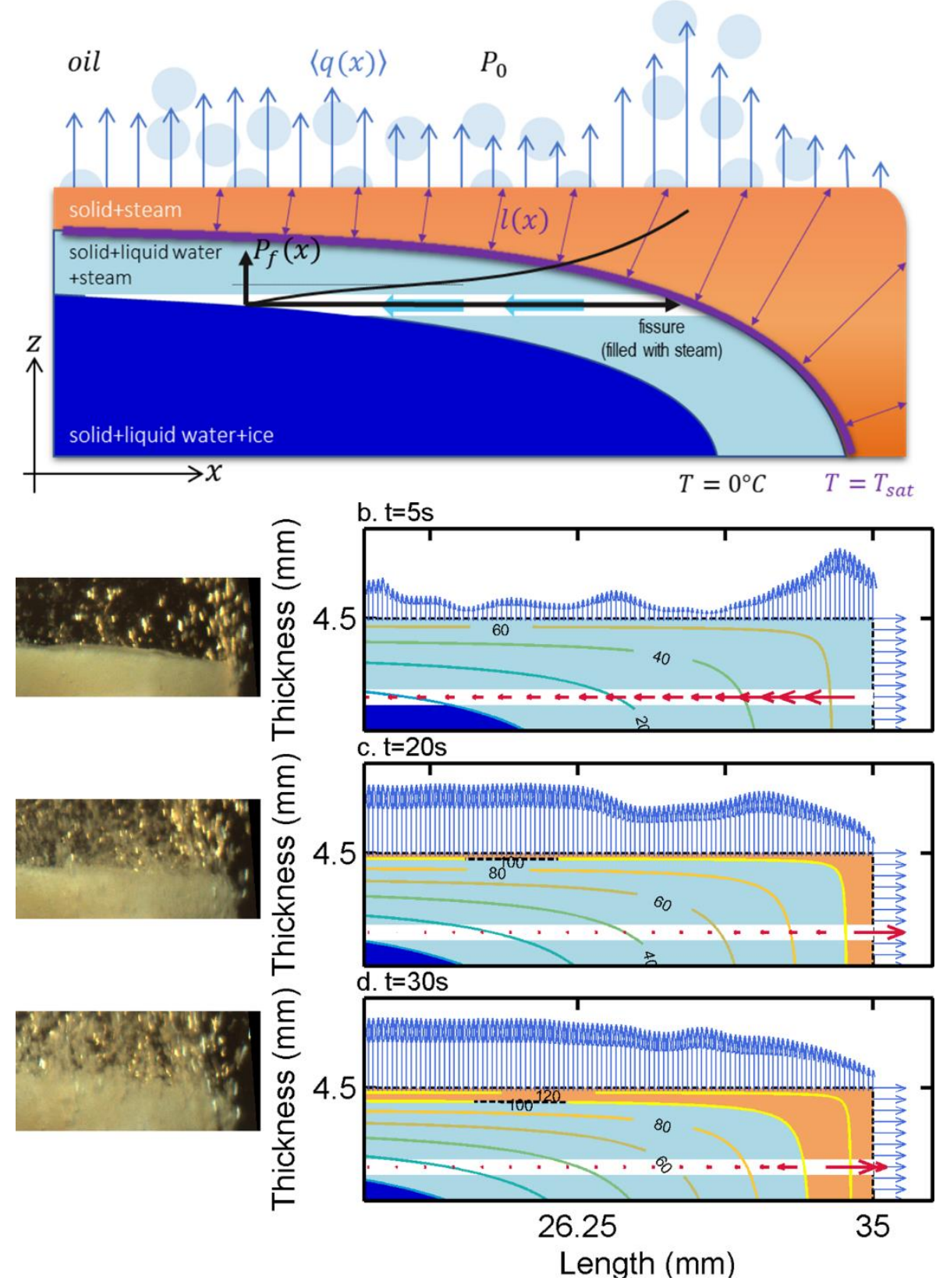

Figure 14. Reconstruction of the longitudinal transport of steam along a hypothetical fissure (white region): (a) main physical assumptions (see Eqs. (2)-(4)), (b-d) reconstruction details based on the bubbling rate profile and isotemperature contours during immersion of parfried frozen samples.

\subsection{Direct observation of oil penetration due to steam condensation}

Due to the volatility of temperature and pressure fields during immersion, it was challenging to prove undoubtedly that steam condensation could cause the penetration of oil in all parts of French-fries (dry or not). A specific configuration with half immersion of the 
parfried frozen product was used to demonstrate the following thermodynamic cycle: i) heat is used to generate steam, ii) steam is transported upwards beyond the immersion line along internal fissures, iii) steam condensate when it meets ice, iv) pressure drop creates a strong suction force accelerating all transports (steam and oil). Results from 18 replicates are shown in Figure 15.

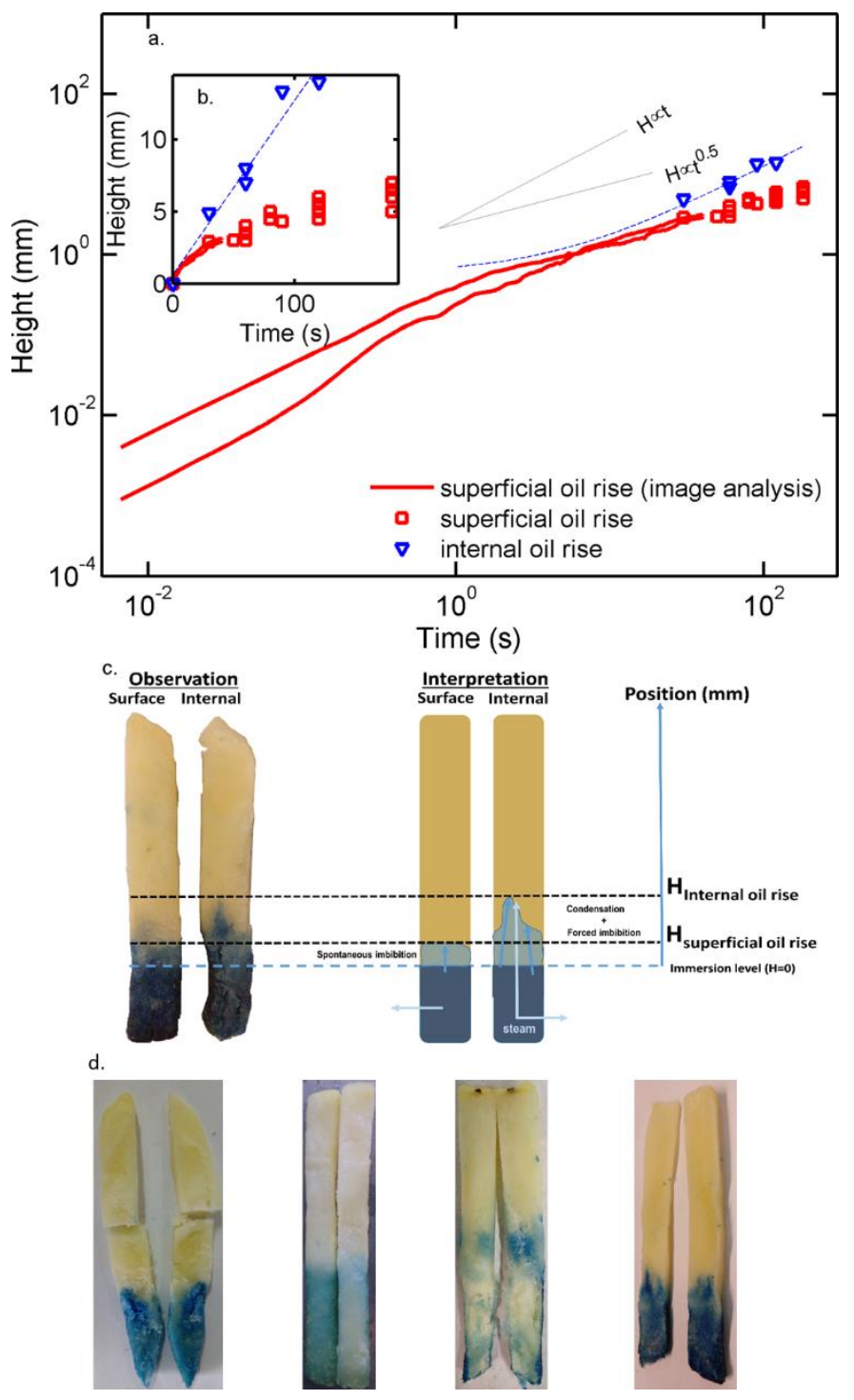

Figure 15. Comparison of the oil rise (dyed in blue) at the surface and inside the product when the initially parfried frozen strip is maintained half-immersed in "blue" oil at $180^{\circ} \mathrm{C}$ : average oil rise above the immersion line on (a) $\log$-log and (b) linear scales; (c) comparison between surface and internal rises; (d) repeated observations of internal rise. 
The studied configuration enabled to extend the coexistence period of ice and steam from $25 \mathrm{~s}$ (in Figure 13) to $100 \mathrm{~s}$. Oil moved vertically along the crust almost instantaneously. During the first $0.1 \mathrm{~s}$, the displacement was almost ballistic (proportional to time). This mechanism corresponded to the mechanism of snap-off at the scale of cells (around $0.1 \mathrm{~mm}$ ). Beyond, the capillary rise was proportional to the square root of time, confirming its spontaneous nature and its control by surface tension effects. Dissections after $10 \mathrm{~s}$ of immersion demonstrated that during the meantime, an internal front moved upwards faster proportionally to time. The internal rise was 2.5 higher than at the surface to reach more than one centimeter after one minute. Internal migration of oil was either almost uniform or located along specific fissures according to the level of injuries in the sample (Figure 15d). The internal forced imbibition observed in these specific experiments were commensurable to the penetration depths observed during the first minute of finish-frying in parfried frozen products (see Figure 7). As the phenomena were similar, the same causes are thought to provoke the same effects in both cases.

\subsection{Description of oil uptake out-of-equilibrium during immersion stage}

This study shows that the mechanisms controlling oil uptake during the immersion stage - when intense vaporization occurs inside parfried frozen product - are determined by the balance between two opposite fates: steam escaping from and condensing inside the frying product. As in bifurcation theory, a small smooth change occurring or made in the food structure may cause a sudden change in both steam and oil flows. It is important noticing that oil and steam do not need to follow the same routes. In Figure 14 and Figure 15, steam follows cracks along the longitudinal direction of the strip (longest dimension), whereas oil percolates through transverse ones (see Figure 8). In this particular case, microscopic flows occur specifically in some defects and are controlled by long-range fluctuations of the pressure field. These 
phenomena cannot be approximated from averaged macroscopic descriptions and thermodynamical considerations (entropy maximization) of saturation and wetting properties. Such rapid evolutions are, indeed, not entropy-driven, and they do not lead to uniform oil imbibition as observed once the product is removed from the oil bath. Similar out-ofequilibrium and brutal behaviors during the immersion stage have been already described for the transport of liquid water inside the hygroscopic crust above the boiling point of free water. Vitrac et al. (2003) and Achir et al. (2009) showed, indeed, that liquid water was frequently ejected from foods with loose structures during frying (i.e., without dense crusts). These outstanding phenomena explain the seldom accumulation of liquid water at the bottom of continuous and batch deep-fryers. The porous structure in parfried products is highly heterogeneous and anisotropic (see Figure 12). The frozen core prevents the homogenization of the internal pressure between both ends of the strip during the minute of frying. As a result, different phenomena can occur on both ends of the parfried frozen strip (i.e. no symmetry plane). In addition, the drying of ends several seconds forward faces affects how steam can circulate inside the product. The transport of steam is aligned with the longitudinal cracks during the first seconds of immersion, before being oriented towards the main faces once the pressure drop across the ends becomes too high. The "impermeabilization" of ends would make possible the redirection of steam towards the frozen core. This scenario is physically possible as demonstrated by the oil uptake pattern of Figure 15 .

\section{Conclusions}

This study confirms that, in most of the cases, oil cannot penetrate during the immersion stage. Parfrying wets the product surface with oil and induces a superficial drying, but it does not change the conclusion. The internal pressure created by the pressure loss across the crust when 
steam escapes the product opposes, indeed, to the oil capillary pressure in the crust. The gradient of pressure reverses, however, during cooling and when ice is present inside parfried frozen product. The latter causes a brutal condensation of steam during the early stages of immersion and destabilizes the internal pressure field. During frying, steam can meet ice only in favor of cracks and fissures, which developed in the longitudinal direction during long-term storage of parfried frozen French-fries. Steam usually flows in the direction of less pressure drop, which is perpendicular to the crust, but a longitudinal flow was shown to be possible and very likely in the very first minute of frying. The concentric and conic shapes of the vaporization and melting fronts close to strip ends make the longitudinal flow from ends towards center more probable than the reverse one. As a result, no "blue oil" was observed in ends. The internal flow of steam cannot alone explain the penetration of oil and should be envisioned as the combined effects of internal steam condensation and oil penetration in a different fissure connecting the external surface obliquely with the frozen region. The existence of a network of cracks with ramifications in the longitudinal and transverse directions has been evidenced by the frequent planar fractures combining oblique fissures in parfried frozen products.

The amount of "blue oil" (oil impregnated during frying) is minor (between $2 \%$ and $6 \%$ of the total oil) and can be avoided easily by thawing French-fries before finish frying. The new insights on the physics of frying brought by these new observations are, however, more significant. Approximatively half of the parfrying oil is lost during finish-frying. That is that the steam flow through the crust prevents not only oil impregnation, but also participates in removing oil absorbed during parfrying. Fissures and defects offered penetration routes only to blue oil and not to red oil. The reason is that the amount of dragged red oil is limited when the product is removed from the bath, and the capillary penetration is fast only in the small pores offered by the size of the cells and the capillaries created in molten starch. The deep penetration of blue oil demonstrated that the core parenchyma cells lost most of their cohesion in the 
conditions of frying after parfrying, frozen storage, ice melting and heating. The situation contrasts dramatically with the fresh potato products where the honeycomb cellular structure is well preserved. Many cracks open at cellular scale as a zip facilitating the circulation of steam inside the product. The strong flow of steam around cells might cause some deviations to nonlocal thermodynamical equilibrium, like the ones related to the circulation of steam towards non-boiling regions (with ice or below the water saturation temperature).

Limiting oil uptake is usually the most common goal. It is essentially a surface phenomenon with $95 \%$ of the oil located either inside the cells or at a wetting film when the product is removed from the bath. The thermal expansion of the parfrying oil combined with its displacement by steam is the likeliest reason for the partial removal of parfrying oil. Similar strategies could be derived to reduce oil uptake or defat finished products. Conversely, if a deep penetration of oil is thought, the use of frozen products partly or fully immersed and with controlled defects can orient the amount and the location of oil. Additionally, since mainly the oil at the surface of the product is absorbed, the entire deep-frying process could be redesigned by using a very thermostable oil during the first minutes of frying and by using a different oil during the very last seconds. Oil with high nutritional value, possibly sensitive to oxidation, could be added during the last seconds of temperature recovery either by immersion or pouring. The details of the dripping process are part of a companion work.

\section{Acknowledgments}

The authors would like to thank Regis Kesteloot for the microtomography imaging.

\section{Funding}


This work was supported by a grant of "Investments d'Avenir" Programme (FUI-AAP17

Fry'In) for the collaborative project Fry'In.

\section{References}

Achir, N., Vitrac, O., Trystram, G., 2009. Heat and mass transfer during frying, Advances in Deep-Fat Frying. CRC Press, Boca-Raton, pp. 5-32.

Achir, N., Vitrac, O., Trystram, G., 2010. Direct observation of the surface structure of French fries by UV-VIS confocal laser scanning microscopy. Food Research International 43, 307314.

Aguilera, J.M., Cadoche, L., López, C., Gutierrez, G., 2001. Microstructural changes of potato cells and starch granules heated in oil. Food Research International 34, 939-947.

Aguilera, J.M., Gloria, H., 1997. Determination of oil in fried potato products by differential scanning calorimetry. Journal of Agricultural and Food Chemistry 45, 781-785.

Benczdi, D., Tomka, I., Escher, F., 1998. Thermodynamics of Amorphous StarchWater Systems. 1. Volume Fluctuations. Macromolecules 31, 3055-3061.

Bennethum, L.S., Cushman, J.H., 1996. Multiscale, hybrid mixture theory for swelling systems - I: balance laws. International Journal of Engineering Science 34, 125-145.

Bouchon, P., Pyle, D.L., 2005a. Modelling Oil Absorption During Post-Frying Cooling: I: Model Development. Food and Bioproducts Processing 83, 253-260.

Bouchon, P., Pyle, D.L., 2005b. Modelling Oil Absorption During Post-Frying Cooling: II: Solution of the Mathematical Model, Model Testing and Simulations. Food and Bioproducts Processing 83, 261-272.

Cortés, P., Badillo, G., Segura, L., Bouchon, P., 2016. The effect of different porous media on moisture loss and oil absorption profiles during frying using glass micromodels. AIChE Journal 62, 629-638.

Dana, D., Saguy, I.S., 2006. Review: Mechanism of oil uptake during deep-fat frying and the surfactant effect-theory and myth. Advances in Colloid and Interface Science 128-130, 267272.

Drewnowski, A., Popkin, B.M., 1997. The nutrition transition: new trends in the global diet. Nutrition reviews 55, 31-43.

Gillet, G., Vitrac, O., Desobry, S., 2011. A fast method to assess the composition of a polyolefin: an application to compliance testing of food contact materials. Journal of Applied Polymer Science 119, 1492 - 1515.

Gormley, R., Walshe, T., Hussey, K., Butler, F., 2002. The Effect of Fluctuating vs. Constant Frozen Storage Temperature Regimes on Some Quality Parameters of Selected Food Products. Food Science and Technology 35, 190-200.

Halder, A., Dhall, A., Datta, A.K., 2007a. An Improved, Easily Implementable, Porous Media Based Model for Deep-Fat Frying: Part I: Model Development and Input Parameters. Food and Bioproducts Processing 85, 209-219.

Halder, A., Dhall, A., Datta, A.K., 2007b. An Improved, Easily Implementable, Porous Media Based Model for Deep-Fat Frying: Part II: Results, Validation and Sensitivity Analysis. Food and Bioproducts Processing 85, 220-230.

Hubbard, L.J., Farkas, B.E., 2000. Influence of Oil Temperature on Convective Heat Transfer During Immersion Frying. Journal of Food Processing and Preservation 24, 143-162. 
Li, J., Ge, W., Wang, W., Yang, N., Huang, W., 2016a. Focusing on mesoscales: from the energy-minimization multiscale model to mesoscience. Current Opinion in Chemical Engineering 13, 10-23.

Li, J., Ge, W., Wang, W., Yang, N., Liu, X., Wang, L., He, X., Wang, X., Wang, J., Kwauk, M., 2016b. From Multiscale Modeling to Meso-Science. Springer, Berlin, Heidelberg.

Mellema, M., 2003. Mechanism and reduction of fat uptake in deep-fat fried foods. Trends in Food Science \& Technology 14, 364-373.

Ni, H., Datta, A.K., 1999. Moisture, Oil and Energy Transport During Deep-Fat Frying of Food Materials. Food and Bioproducts Processing 77, 194-204.

Patsioura, A., Vauvre, J.-M., Kesteloot, R., Jamme, F., Hume, P., Vitrac, O., 2015. Microscopic imaging of biphasic oil-air flow in French fries using synchrotron radiation. AIChE Journal 61, 1427-1446.

Patsioura, A., Vauvre, J.M., Kesteloot, R., Smith, P., Trystram, G., Vitrac, O., 2016. Mechanisms of Oil Uptake in French Fries, in: Singh, J., Lovedeep, K. (Eds.), Advances in Potato Chemistry and Technology, 2nd ed. Academic Press, 125 London Walln London EC2Y 5AS, UK, pp. 503-526.

Pedreschi, F., 2012. Frying of Potatoes: Physical, Chemical, and Microstructural Changes. Drying Technology 30, 707-725.

Sandhu, J., Bansal, H., Takhar, P.S., 2013. Experimental measurement of physical pressure in foods during frying. Journal of Food Engineering 115, 272-277.

Sandhu, J.S., Takhar, P.S., 2018. Verification of hybrid mixture theory based two-scale unsaturated transport processes using controlled frying experiments. Food and Bioproducts Processing 110, 26-39.

Ullah, J., Takhar, P.S., Sablani, S.S., 2014. Effect of temperature fluctuations on ice-crystal growth in frozen potatoes during storage. LWT - Food Science and Technology 59, 1186-1190. Vauvre, J.M., Patsioura, A., Kesteloot, R., Vitrac, O., 2015. Multiscale modeling of oil uptake in fried products. Aiche Journal 61, 2329-2353.

Vitrac, O., Dufour, D., Trystram, G., Raoult-Wack, A.-L., 2002. Characterization of heat and mass transfer during deep-fat frying and its effect on cassava chip quality. Journal of Food Engineering 53, 161-176.

Vitrac, O., Raoult-Wack, A.L., Trystram, G., 2003. Influence of Liquid Water Transport on Heat and Mass Transfer during Deep-Fat-Frying, in: Welti-Chanes, J., Vélez-Ruiz, J.F., Barbosa-Canovas, G.V. (Eds.), Transport phenomena in food processing CRC Press, BocaRaton, USA, pp. 445-462.

Vitrac, O., Trystram, G., 2005. A method for time and spatially resolved measurement of convective heat transfer coefficient (h) in complex flows. Chemical Engineering Science 60, 1219-1236.

Vitrac, O., Trystram, G., Raoult-Wack, A.L., 2000. Deep-fat frying of food: heat and mass transfer, transformations and reactions inside the frying material. European Journal of Lipid Science and Technology 102, 529-538.

Whitaker, S., 1977. Simultaneous heat, mass, andmomentum transfer in porous media: a theory of drying., in: Hartnett James, P., IrvineThomas, F. (Ed.), Advances in heattransfer. Elsevier, pp. 119-203.

Ziaiifar, A.M., Achir, N., Courtois, F., Trezzani, I., Trystram, G., 2008. Review of mechanisms, conditions, and factors involved in the oil uptake phenomenon during the deep-fat frying process. International Journal of Food Science \& Technology 43, 1410-1423. 